\title{
The transcription factor ERG recruits CCR4-NOT to control mRNA decay and mitotic progression
}

\author{
Xavier Rambout ${ }^{1,2}$, Cécile Detiffe ${ }^{1,2}$, Jonathan Bruyr ${ }^{1,2}$, Emeline Mariavelle $^{1,2}$, Majid Cherkaoui ${ }^{1,2}$, \\ Sylvain Brohée ${ }^{3,4}$, Pauline Demoitié ${ }^{1,2}$, Marielle Lebrun ${ }^{1,5}$, Romuald Soin ${ }^{6}$, Bart Lesage ${ }^{7}$, Katia Guedri ${ }^{1,2}$, \\ Monique Beullens ${ }^{7}$, Mathieu Bollen ${ }^{7}$, Thalia A Farazi ${ }^{8}$, Richard Kettmannn ${ }^{1,2}$, Ingrid Struman ${ }^{1,9}$, David E Hill ${ }^{10,11}$, \\ Marc Vidal $^{10,11}$, Véronique Kruys ${ }^{6}$, Nicolas Simonis ${ }^{3}$, Jean-Claude Twizere ${ }^{1,2}$ \& Franck Dequiedt ${ }^{1,2}$
}

\begin{abstract}
Control of mRNA levels, a fundamental aspect in the regulation of gene expression, is achieved through a balance between mRNA synthesis and decay. E26-related gene (Erg) proteins are canonical transcription factors whose previously described functions are confined to the control of mRNA synthesis. Here, we report that ERG also regulates gene expression by affecting mRNA stability and identify the molecular mechanisms underlying this function in human cells. ERG is recruited to mRNAs via interaction with the RNA-binding protein RBPMS, and it promotes mRNA decay by binding CNOT2, a component of the CCR4-NOT deadenylation complex. Transcriptome-wide mRNA stability analysis revealed that ERG controls the degradation of a subset of mRNAs highly connected to Aurora signaling, whose decay during $\mathbf{S}$ phase is necessary for mitotic progression. Our data indicate that control of gene expression by mammalian transcription factors may follow a more complex scheme than previously anticipated, integrating mRNA synthesis and degradation.
\end{abstract}

Although undisputable evidence has clearly demonstrated that the nuclear steps of mRNA processing are mechanistically linked to transcription, a conceptual evolution in gene regulation has come with the realization that transcription might also be functionally connected to more remote processes occurring in the cytoplasm, such as mRNA decay $^{1,2}$. Cytoplasmic mRNA decay is initiated by deadenylation, a rate-limiting event during which the poly $(\mathrm{A})$ tail of the transcript is trimmed off by the CCR4-NOT complex, the main deadenylation machinery in eukaryotes ${ }^{3}$. The degradation of specific mRNAs, a key process in the regulation of eukaryotic gene expression, is achieved through the recruitment of the CCR4-NOT complex by sequence-specific RNA-binding proteins (RBPs) or by the microRNA machinery ${ }^{3,4}$. Poly(A)-shortened mRNAs, along with factors involved in the deadenylation, decapping and mRNA-degradation machineries, accumulate in microscopic mRNA-protein complex (mRNP) aggregates called processing bodies $(\mathrm{PBs})^{5}$.

The idea of coupling between mRNA synthesis and degradation has recently emerged. Genome-wide expression studies in yeast have shown that mRNA synthesis and decay are mechanistically and functionally coordinated, thus supporting the existence of common molecular effectors $^{6-9}$. In particular, the CCR4-NOT deadenylation complex was first described as a transcriptional regulator and has been implicated in initiation and elongation by RNA polymerase $\mathrm{II}^{10,11}$. More surprisingly, it has also been shown that degradation of yeast mRNAs is determined by cis-acting sequence elements in promoters ${ }^{12,13}$. These findings have led to the concept of mRNA imprinting, in which sequence-specific DNA-binding factors might orchestrate mRNA synthesis and decay. This decay would occur via loading of factors regulating cytoplasmic mRNA degradation onto the transcribing mRNA ${ }^{14}$. However, the identity of these DNA-binding mRNA coordinators is still obscure, and it remains to be tested whether such coupling between transcription and decay also exists in higher eukaryotes.

E26 (Ets) proteins, a family of 28 helix-loop-helix transcription factors (TFs) in metazoans, are characterized by a highly conserved DNA-binding ETS domain ${ }^{15}$. Through this domain, Ets factors bind specific gene promoters and act as key regulators in many biological processes including cellular proliferation, apoptosis, differentiation and survival ${ }^{16}$. ERG, FLI1 and the more structurally divergent FEV compose the Erg subfamily of Ets factors and have been identified as driving factors in prostate cancer, Ewing's tumors and leukemias ${ }^{15,17}$. Using ERG as a paradigm, we sought to investigate the possibility that eukaryotic transcription factors might be directly involved in cytoplasmic mRNA decay. We demonstrate that ERG triggers degradation of mRNAs connected to Aurora signaling by recruiting RBPs and the CCR4-NOT deadenylation complex and that this activity is important for normal mitotic progression.

\footnotetext{
${ }^{1}$ Interdisciplinary Cluster for Applied Genoproteomics (GIGA-R), University of Liège (ULg), Liège, Belgium. ${ }^{2}$ GIGA-Molecular Biology in Diseases, ULg, Liège, Belgium. ${ }^{3}$ BiGRe, Université Libre de Bruxelles (ULB), Bruxelles, Belgium. ${ }^{4}$ Computer Science Department, ULB, Bruxelles, Belgium. ${ }^{5}$ GIGA-Inflammation, Infection \& Immunity, ULg, Liège, Belgium. ${ }^{6}$ Faculté des Sciences, ULB, Gosselies, Belgium. ${ }^{7}$ Department of Cellular and Molecular Medicine, University of Leuven (KUL), Leuven, Belgium. ${ }^{8}$ Howard Hughes Medical Institute, Rockefeller University, New York, New York, USA. ${ }^{9}$ GIGA-Cancer, ULg, Liège, Belgium. ${ }^{10}$ Center for Cancer Systems Biology (CCSB), Department of Cancer Biology, Dana-Farber Cancer Institute (DFCI), Boston, Massachusetts, USA. ${ }^{11}$ Department of Genetics, Harvard Medical School, Boston, Massachusetts, USA. Correspondence should be addressed to F.D. (fdequiedt@ulg.ac.be).
} 


\section{RESULTS}

ERG localizes to PBs and promotes cytoplasmic mRNA decay Initially, we asked whether the subcellular localization of ERG might be compatible with a potential function in cytoplasmic mRNA decay. To answer this question, we examined the localization of endogenous ERG in HeLa cells, which do not express FLI1 or FEV ${ }^{18}$. ERG localized in the nucleus, consistently with its role as a canonical TF, but also localized in the cytoplasm, where it appeared as discrete foci (Fig. 1a). We hypothesized that these ERG cytoplasmic foci might be PBs. Indeed, we found that these foci exactly colocalized with decapping protein1 A (DCP1A) and enhancer of mRNA decapping 4 (EDC4, HEDLS or GE-1), two PB markers ${ }^{19}$ (Fig. 1a and Supplementary Fig. 1a). Similarly to what has been shown for PBs, cycloheximide treatment led to the disappearence of ERG foci (Fig. 1a). In addition to the colocalization of ERG in PBs, YFP-tagged versions of the two other Erg subfamily members FLI1 and FEV also colocalized with RFP-tagged DCP1A (Supplementary Fig. 1b). The localization of ERG in PBs prompted us to test whether ERG might associate with mRNA. To discriminate between proteins directly or indirectly interacting with mRNA, we irradiated cells with UV to covalently cross-link direct RNA-protein interactions, or treated the cells with formaldehyde to fix large protein complexes onto mRNA, and then isolated poly(A)+ mRNPs through an oligo(dT) purification approach ${ }^{20,21}$. The observation that ERG was exclusively retained on the oligo(dT)-bound fraction from formaldehyde-treated cells indicated that ERG is able to associate with mRNP complexes but not to directly bind mRNA (Fig. 1b). RBM9, a well described $\mathrm{RBP}^{22}$, and GAPDH, which has no known mRNP-related function were used as a positive and negative control, respectively.

We next asked whether ERG might also be found in stress granules (SGs), another type of cytoplasmic granule that forms under stress and exchanges mRNPs with $\mathrm{PBs}^{23}$. We subjected HeLa cells to oxidative stress (through arsenite treatment), osmotic stress (through sorbitol treatment) or heat-shock stress and visualized SGs and PBs by staining for RasGAP SH3 domain binding protein (G3BP $)^{24}$ and DCP1A, respectively. ERG independently colocalized with both DCP1A and
Figure 1 ERG localizes in PBs and promotes decay of tethered mRNAs. (a) Immunofluorescence of ERG (green), DPC1A (PB marker, red) and Hoechst (nuclear stain, blue) in either untreated or cycloheximide-treated HeLa cells. Scale bars, $10 \mu \mathrm{m}$. (b) Oligo(dT) pulldown and anti-ERG, anti-RBM9 and anti-GAPDH western blotting (WB). Samples are untreated or RNase A-treated lysates from HeLa cells either crosslinked with UV or fixed with formaldehyde (PAF). Uncropped blots are shown in Supplementary Data Set 1. (c) Immunofluorescence of ERG (green), DPC1A (PB marker, red), G3BP (SG marker, white) and Hoechst (nuclear stain, blue) in arsenite-, sorbitol- or heatshock-treated HeLa cells. Scale bars, $10 \mu \mathrm{m}$. (d) Immunofluorescence of DPC1A (PB marker, white), G3BP (SG marker, white) and Hoechst (nuclear stain, blue) in HeLa cells transfected with either SiCTL or siERG and either left untreated (DCP1A labeling) or treated with arsenite (G3BP labeling). Scale bars, $10 \mu \mathrm{m}$. (e) Quantitative analysis of the number of PBs or SGs per cell from immunofluorescence analysis in $\mathbf{d}$. Results are shown as means \pm s.d. $(n=7$ independent experiments with $100-150$ cells counted in each replicate). ${ }^{* * *} P<0.001$ compared to SiCTL by two-tailed unpaired Student's $t$ test. (f) RT-qPCR analysis of the stability of the R-LuC-8MS2 and the $R$-LuC-OMS2 mRNA reporters. Samples are RNA from HeLa cells transfected with the indicated R-Luc reporters and either ERG-MS2-CP or MS2-CP, and treated for $0,1,2$ or $4 \mathrm{~h}$ with ActD. Results are shown as means \pm s.d. ( $n=5$ and 3 independent experiments for $R$-LuC-8MS2 and $R$-Luc-OMS2, respectively). ${ }^{*} P<0.05$; ${ }^{* *} P<0.01 ; * * * P<0.001$; NS, not significant compared to MS2-CP by two-tailed unpaired Student's $t$ test. $t_{1 / 2}$, half-life. (g) RT-qPCR analysis of the stability of the R-Luc-8MS2 mRNA reporter. Samples are RNA from HeLa cells transfected with siCTL or anti-GW182 siRNA (siGW182), with ERG-MS2-CP or MS2-CP, with the R-Luc-8MS2 reporter, and treated for 0, 1, 2 or $4 \mathrm{~h}$ with ActD. Results are shown as means \pm s.d. ( $n=4$ independent experiments). ${ }^{*} P<0.05 ;{ }^{*} P<0.01$; NS, not significant compared to siCTL by two-tailed unpaired Student's $t$ test. Source data for $\mathbf{e}-\mathbf{g}$ are available online. a ERG
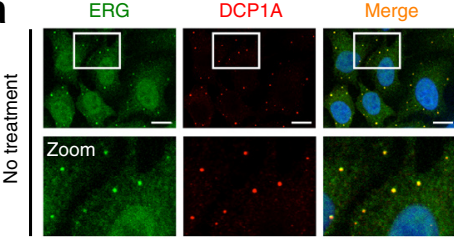

C
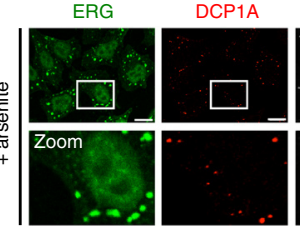

G3BP
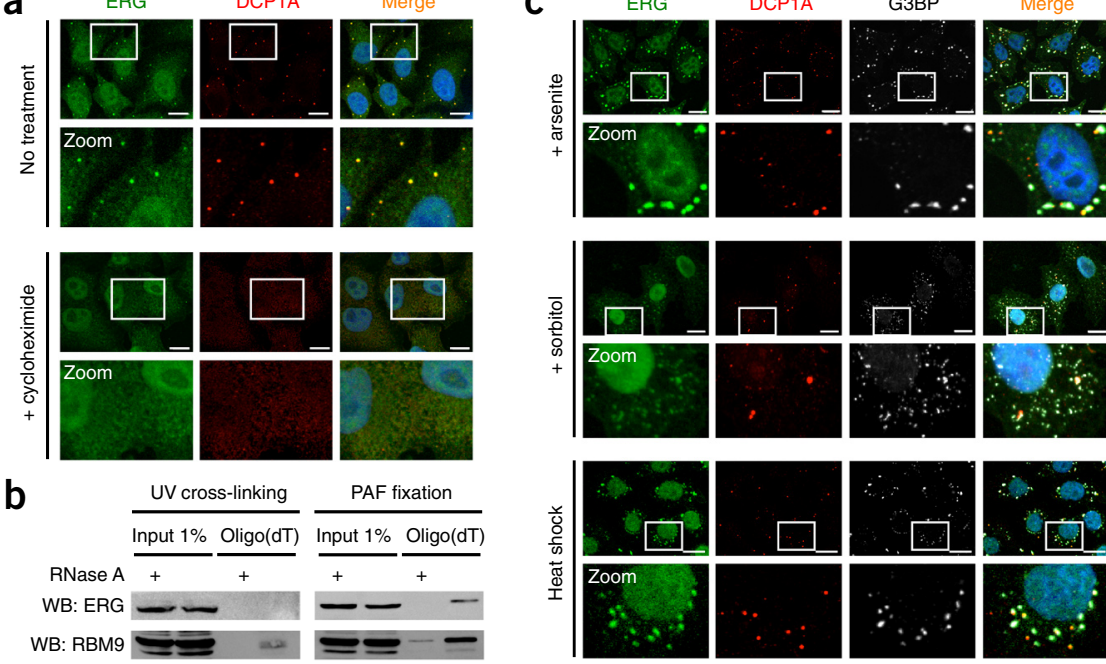

b
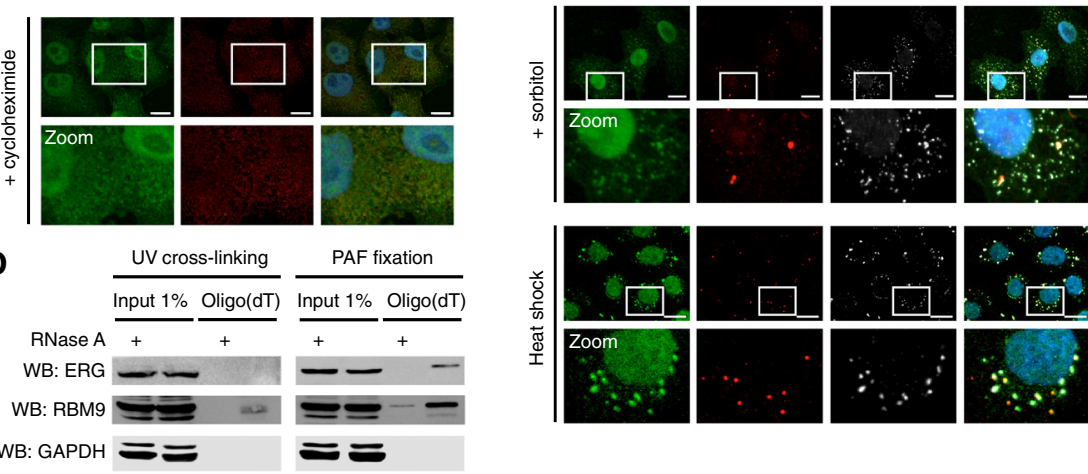

d
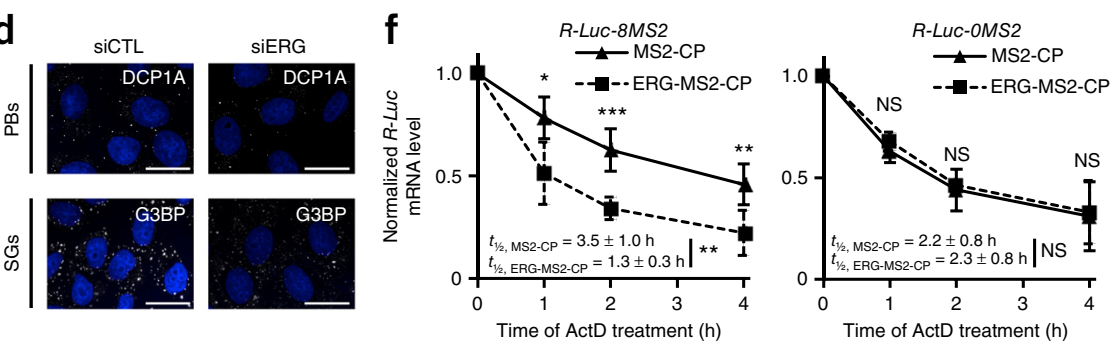

e

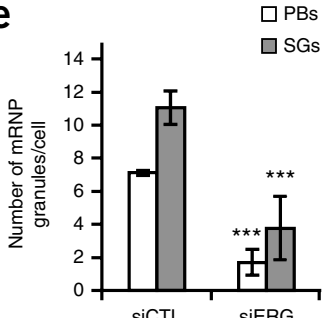

g
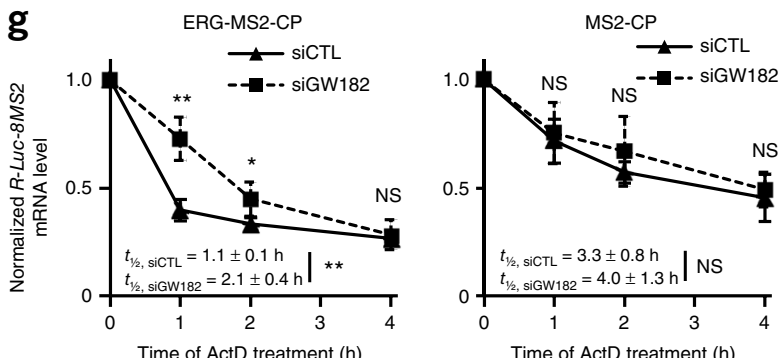

Time of ActD treatment (h) 
G3BP, thus indicating that it belongs to the set of common components shared between PBs and SGs (Fig. 1c). Notably, ERG also colocalized with T-cell intracellular antigen-1 (TIA1) and TIA1-related protein (TIAR), two additional SG markers ${ }^{25}$ (Supplementary Fig. 1c). Finally, ERG knockdown (Supplementary Fig. 1d,e) led to a significant reduction in the number of both PBs and SGs (Fig. 1d,e), thus establishing ERG as a key component of mRNP complexes cycling between PBs and SGs and supporting its role in mRNA degradation.

On the basis of these observations, we tested the function of ERG in mRNA decay by using a previously described tethering assay ${ }^{26}$. This procedure uses a Renilla luciferase reporter mRNA carrying eight repeats of the binding sequence for the bacteriophage MS2 coat protein (MS2-CP) in its $3^{\prime}$ untranslated region (denoted $R$-Luc-8MS2), thus allowing specific recruitment of any protein of interest fused to the MS2-CP peptide. We transfected FLAG-tagged constructs encoding either MS2-CP alone or MS2-CP fused with ERG (ERGMS2-CP) together with the R-Luc-8MS2 reporter or with a control reporter lacking the MS2 binding sites (denoted R-Luc-OMS2). For standardization, the R-Luc-8MS2 and R-Luc-OMS2 constructs expressed firefly luciferase (F-Luc) and Renilla luciferase from the same bidirectional promoter. Recruitment of ERG to the R-Luc transcript specifically reduced the expression of the R-Luc protein in two cancerous cell lines (HeLa and U2OS) and one noncancerous cell line (MRC5). Quantitative mRNA analysis revealed that ERG inhibited R-Luc activity predominantly by reducing the abundance of the tethered $R$-Luc-8MS2 transcript (Supplementary Fig. 1f). We next assessed the decay rates of the R-Luc-8MS2 and R-Luc-OMS2 mRNAs in HeLa cells by RT-qPCR under conditions of transcriptional blockage by actinomycin D (ActD). Compared with MS2-CP expression, the expression of ERG-MS2-CP decreased the stability of the $R-L u c-8 M S 2$ reporter mRNA but had no effect on the untethered $R$-Luc-OMS 2 mRNA (Fig. 1f). The ability of ERG to promote mRNA degradation appeared to be independent of its transcriptional activity, because a transcriptionally defective mutant of ERG (W334R ${ }^{27}$ ) was as efficient as wild-type (WT) ERG in inducing degradation of the a
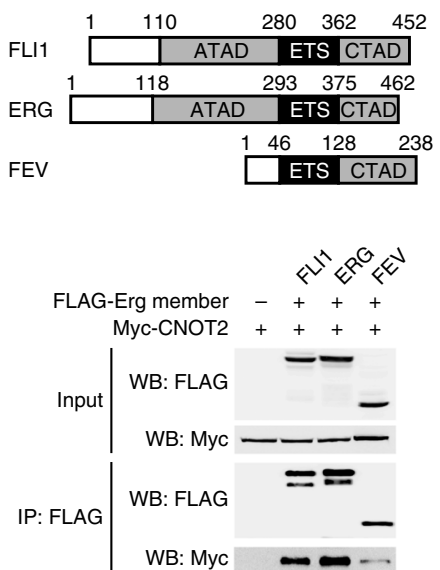

b

\begin{tabular}{|c|c|c|}
\hline & 118 & $293 \quad 375 \quad 46$ \\
\hline ERG-FL & ATAD & ETS CTAD \\
\hline ERG $\triangle A T A D$ & & ETS CTAD \\
\hline ERG $\Delta$ ETS & ATAD & TAD \\
\hline ERG $\triangle C T A D$ & ATAD & \\
\hline
\end{tabular}

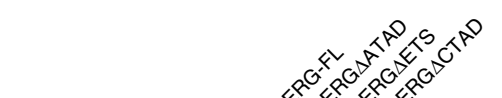

FLAG-ERG mutant -++++ Myc-CNOT2
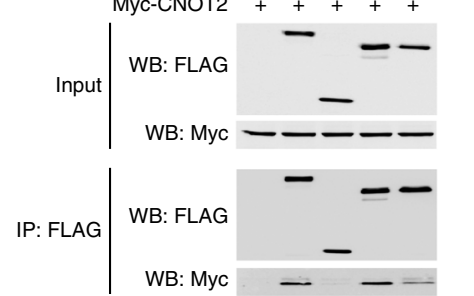

C

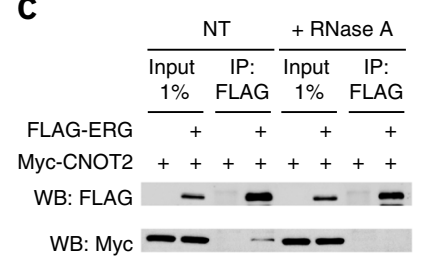

e Input $1 \%+$ IP: CTL IP: ERG WB: ERG WB: CNOT2 WB: CNOT3 WB: CNOT7 d
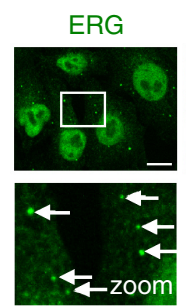
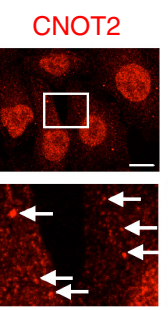

DCP1A

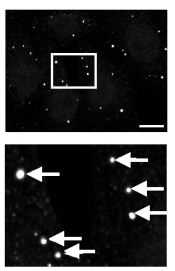

Merge

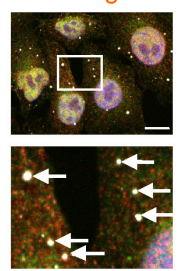

f
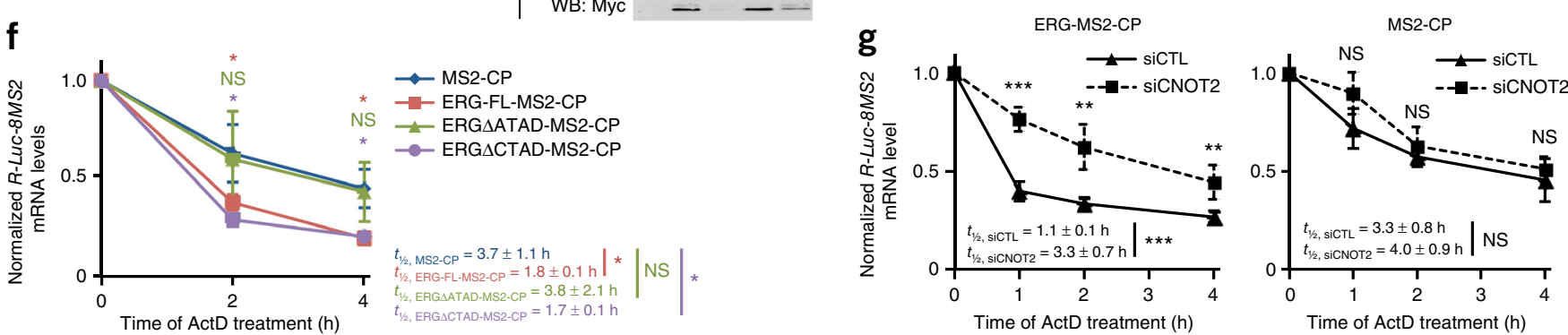

Figure 2 ERG interacts with the CNOT2 component of the CCR4-NOT deadenylation complex in inducing mRNA decay. (a,b) Schematic, domain structure of the Erg subfamily members (a) or ERG-deletion mutants (b). Immunoprecipitation (IP) of FLAG-tagged Erg members (a) or ERG-deletion mutants (b) and anti-FLAG and anti-Myc western blotting. Samples are from HEK-293 cells transfected with Myc-CNOT2 alone or with either of the FLAG-tagged Erg members (a) or ERG-deletion mutants (b). Uncropped blots are shown in Supplementary Data Set 1. FL, full length.

(c) Immunoprecipitation of FLAG-tagged ERG and anti-FLAG and anti-Myc western blotting. Samples are nontreated (NT) or RNase A-treated lysates from HEK-293 cells transfected with Myc-CNOT2 alone or together with FLAG-ERG. Uncropped blots are shown in Supplementary Data Set 1.

(d) Immunofluorescence of ERG (green), CNOT2 (red), DPC1A (PB marker, white) and Hoechst (nuclear stain, blue) in HeLa cells. Scale bars, $10 \mu$ m. (e) Immunoprecipitation of ERG and anti-ERG, anti-CNOT2, anti-CNOT3 and anti-CNOT7 western blotting. Samples are control (CTL) or anti-ERG immunoprecipitates from HeLa cells. Uncropped blots are shown in Supplementary Data Set 1. (f) RT-qPCR analysis of the stability of the R-Luc-8MS2 mRNA reporter. Samples are RNA from HeLa cells transfected with MS2-CP (blue diamonds), ERG-FL-MS2-CP (red squares), ERG $\triangle A T A D-M S 2-C P$ (green triangles) or ERG $\triangle$ CTAD-MS2-CP (purple circles), together with the R-Luc-8MS2 reporter, and treated with ActD for 0, 2 or 4 h. Results are shown as means \pm s.d. ( $n=3$ independent experiments). ${ }^{*} P<0.05$; NS, not significant compared to MS2-CP by two-tailed unpaired Student's $t$ test. (g) RT-qPCR analysis of the stability of the R-Luc-8MS2 mRNA reporter. Samples are RNA from HeLa cells transfected with siCTL or anti-CNOT2 siRNA (siCNOT2), ERG-MS2-CP or MS2-CP, and the R-Luc-8MS2 reporter, and treated with ActD for $0,1,2$ or $4 \mathrm{~h}$. Results are shown as means $\pm \mathrm{s} . \mathrm{d}$. ( $n=4$ independent experiments). ${ }^{* *} P<0.01 ;{ }^{* *} P<0.001$; NS, not significant compared to siCTL by two-tailed unpaired Student's $t$ test. Source data for $\mathbf{f}$ and $\mathbf{g}$ are available online. 


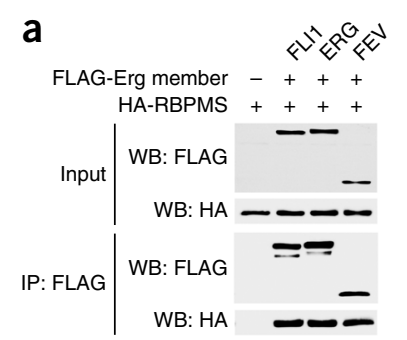

Figure 3 ERG is recruited to $\mathrm{mRNA}$ through its interaction with RBPMS. (a) Immunoprecipitation of FLAG-tagged Erg members and anti-FLAG and anti-HA western blotting. Samples are lysates from HEK-293 cells transfected with HA-RBPMS alone or with either of the FLAG-tagged Erg members. Uncropped blots are shown in Supplementary Data Set 1. (b) Schematic representation of the RIP experiment. Bar graph, RT-qPCR analysis of GAPDH and R-Luc-8MS2 mRNAs. Western blots, anti-FLAG and anti-HA western blots. Samples are lysates from HEK-293 cells transfected with the R-Luc-8MS2 reporter together with HA-tagged RBPMS-MS2-CP or FLAG-ERG alone or in combination. Levels of $R$-Luc-8MS2 mRNA in anti-FLAG immunoprecipitates are expressed as a percentage of the corresponding inputs, normalized to those of GAPDH and set to 1 in the control condition. Results are shown as means \pm s.d. ( $n=3$ independent experiments). ${ }^{*} P<0.05$; NS, not significant compared to control by two-tailed unpaired Student's $t$ test. Uncropped blots are shown in Supplementary Data Set 1 . Source data for $\mathbf{b}$ are available online. (c) Immunofluorescence of ERG (green), RBPMS (red), DPC1A (PB marker, white) and Hoechst (nuclear stain, blue) in HeLa cells. Scale bars, $10 \mu \mathrm{m}$. White and red arrows indicate ERG-containing PBs positive or negative for RBPMS, respectively. (d) Immunoprecipitation of HA-RBPMS and anti-Myc, anti-FLAG and anti-HA western blotting. Samples are lysates from HEK-293 cells transfected with Myc-CNOT2 together with FLAG-ERG or HA-RBPMS alone or in combination. Uncropped blots are shown in Supplementary Data Set 1. (e) Sequential immunoprecipitation of FLAG-ERG and HA-RBPMS, and anti-Myc, anti-FLAG and anti-HA western blotting. Samples are lysates from HEK-293 cells transfected with Myc-CNOT2 together with FLAG-ERG or HA-RBPMS alone or in combination. Uncropped blots are shown in Supplementary Data Set 1.

tethered reporter transcript (Supplementary Fig. 1g,h). Finally, ERGmediated mRNA degradation relied on the presence of intact PBs within the cell, because preventing their formation with a short interfering RNA (siRNA) against the key PB component GW182 (ref. 28) significantly reduced degradation of the R-Luc-8MS2 mRNA by ERG (Fig. 1g and Supplementary Fig. 1i,j). Altogether, these analyses demonstrated that ERG promotes mRNA degradation when it is recruited to specific transcripts.

ERG promotes mRNA decay through recruitment of CCR4-NOT To dissect the molecular mechanisms underlying our findings, we performed high-throughput yeast-two hybrid (HT-Y2H) screens. Using a stringent experimental framework described previously, we tested the full-length versions and the isolated functional domains of the three Erg members (Fig. 2a) against the 15,483 open reading frames (ORFs) of the human ORFeome v5.1 (ref. 29). We identified 28 high-confidence Erg-interacting partners (Supplementary Table 1), which included CNOT2, a component of the CCR4-NOT complex ${ }^{30}$. Interestingly, in the context of TF-mediated mRNA decay, CNOT2 can associate with chromatin in the nucleus and with PBs in the cytoplasm $^{30,31}$. To validate these findings, we performed coimmunoprecipitations between FLAG-tagged Erg members and Myc-CNOT2 and found that CNOT2 coimmunoprecipitated efficiently with ERG and FLI1 but poorly with FEV, which lacks an ATAD (Fig. 2a). When testing the ability of ERG deletion mutants to associate with CNOT2, we observed comparable amounts of CNOT2 copurifying with the full-length ERG protein or with a mutant lacking the central ETS domain, thus indicating that this DNA-binding domain is dispensable for CNOT2 interaction (Fig. 2b). The mutant lacking the carboxy-terminal transcriptional activation domain (CTAD) still interacted with CNOT2, although this interaction was impaired. In contrast, the absence of the amino-terminal transcriptional activation domain (ATAD) rendered ERG unable to copurify with CNOT2, thus confirming the importance of this domain in the ERGCNOT2 interaction. Using CNOT2-deletion mutants we found that removal of the Not1 anchor region (NAR, amino acids 350-408) of CNOT2 greatly impaired but did not abolish interaction with $\mathrm{ERG}^{32}$ (Supplementary Fig. 2a). We also found that treatment of cell lysates with RNase A dramatically reduced interaction between ERG and CNOT2, thus indicating that formation of a stable ERG-CNOT2 complex relies on RNA and suggesting that both proteins are part of the same mRNPs (Fig. 2c). Consistently with this idea, endogenous ERG colocalized with CNOT2 in both PBs (Fig. 2d) and SGs (Supplementary Fig. 2b).

ERG also interacted with CNOT3, a direct partner of CNOT2 in CCR4-NOT ${ }^{32}$ (Supplementary Fig. 2c). Together, CNOT2 and CNOT3 form the NOT module, which is thought to mediate the recruitment of the deadenylase subunits of the CCR4-NOT complex to mRNAs targeted for degradation ${ }^{3,33}$. Indeed, ERG also coimmunoprecipitated with both deadenylase subunits CNOT6 (CCR4) and CNOT7 (CAF1), a result consistent with a function of ERG in mRNA decay (Supplementary Fig. 2c). Importantly, we also confirmed interactions between ERG and components of the NOT and deadenylase modules of CCR4-NOT on endogenous proteins (Fig. 2e).

On the basis of these observations, we speculated that ERG-mediated mRNA decay would rely directly on CNOT2. In agreement with this 
Figure 4 ERG, RBPMS and CNOT2 promote decay of mitotic mRNAs. (a) Scatter plot of mRNA stabilities in HeLa cells transfected with either siCTL or siERG and treated with ActD for $0,0.5,1,2$ or $4 \mathrm{~h}$. For each mRNA, the area under the decay curve in the SiCTL condition is plotted against the area under the decay curve in the siERG condition. Dashed lines are the significance limits of the fitted normal distribution curve of the area differences (details in Online Methods). Green and red dots are mRNAs that are the most significantly stabilized or degraded, respectively, after ERG knockdown. Decay curves were calculated from means of four independent experiments. (b) Overlay between the 186 ERG mRNAdegradation targets (green dots in a) and the 4,839 confident RBPMS-interacting mRNAs identified in ref. 36 and showing a negative decay slope in siCTL-transfected HeLa cells. The $P$ value was determined by Pearson's chisquared test. (c) Cumulative distribution of the mRNA decay rates of nontargets (blue line) or PAR-CLIP RBPMS targets (red line ref. 36) determined from ERG-knockdown HeLa cells (as described in a). The $P$ value was calculated by Kolmogorov-Smirnov test. (d) RT-qPCR decay analysis of the 22 ERG mitotic targets (identified in Supplementary Fig. 4a). Samples are RNA from HeLa cells transfected with siCTL, siERG, siCNOT2 or anti-RBPMS siRNA (siRBPMS) and treated for $0,1,2$ or $4 \mathrm{~h}$ with ActD ( $n=5,4,3$ and 4 independent experiments, respectively). The mean half-life of each mitotic target in control cells is plotted against its mean half-life in siERG-, siCNOT2- or siRBPMS-treated cells. Upper and lower dashed lines indicate the 1.5-fold increase and decrease in half-life, respectively, compared with control cells. For clarity, s.d. are not shown but are displayed in Supplementary Figure 4b. Source data for this figure are available online. a

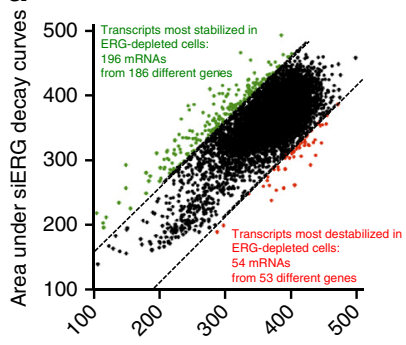

Area under siCTL decay curves

d

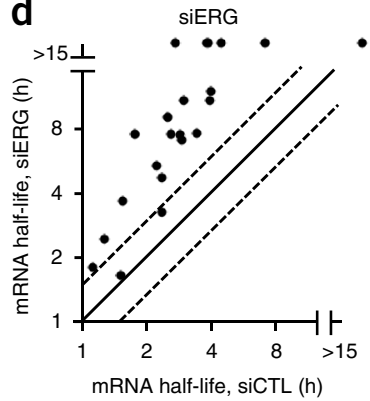

b
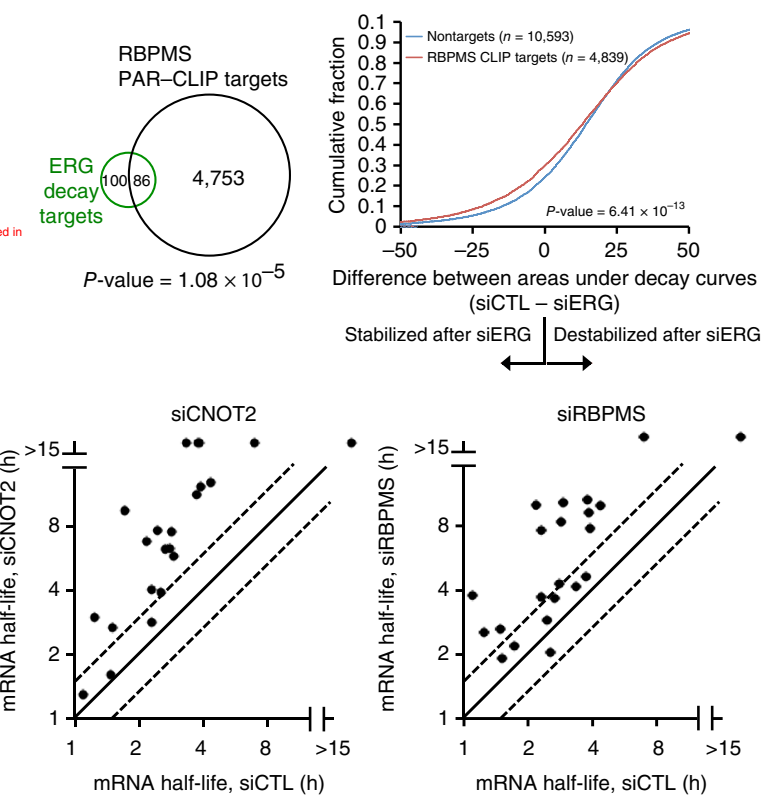

possibility, we observed that tethering of the $\triangle$ ATAD ERG mutant, which lacks the ability to interact with CNOT2 (Fig. 2b), had no effect on the stability of the $R$-Luc-8MS2 reporter mRNA (Fig. $2 \mathbf{f}$ and Supplementary Fig. 2d). In addition, knockdown of CNOT2 prevented ERG-mediated degradation of the $R$-Luc-8MS2 reporter mRNA (Fig. 2 g and Supplementary Fig. 2e,f) without affecting basal degradation of R-Luc-8MS2 (i.e., in the absence of ERG-MS2-CP tethering). Altogether, these results provide strong evidence that ERG triggers the degradation of bound mRNAs through CNOT2 and recruitment of the CCR4-NOT deadenylation complex.

\section{ERG is recruited to mRNA via its interaction with RBPs}

Next, we addressed the critical question of how ERG might be recruited to mRNA. Strikingly, the set of ERG interactors identified in our Y2H screen also included RNA-binding Fox-1 (RBFOX1 or A2BP1) and RNA-binding protein with multiple splicing (RBPMS or Hermes), two RBPs with canonical RNA-recognition motifs ${ }^{34,35}$ (Supplementary Table 1). Whereas RBFOX proteins are wellcharacterized splicing factors, the role of RBPMS in mRNA processing remains unclear ${ }^{36,37}$. From these findings, we hypothesized that ERG might be recruited to specific mRNAs through its interaction with RBPs including RBPMS. Coimmunoprecipitation experiments with ectopically expressed proteins revealed that Erg family members share the ability to associate with RBPMS, thus supporting our model (Fig. 3a). Importantly, we also confirmed the existence of a physiological ERG-RBPMS interaction by coimmunoprecipitation of endogenous proteins from HeLa cell extracts (Supplementary Fig. 3a).

By using ERG-deletion mutants, we showed that the CTAD is critical for interaction with RBPMS (Supplementary Fig. 3b). The observations that the ERG-RBPMS interaction did not rely on the presence of RNA (Supplementary Fig. 3c) and that an RBPMS mutant lacking the RNA-recognition motif still interacted with ERG (Supplementary Fig. 3d) suggested that binding to mRNA and association with ERG involve separate domains of RBPMS and are thus compatible with a model in which RBPMS recruits ERG to mRNAs. To test this possibility, we performed indirect RNA immunoprecipitation (RIP) experiments in the context of the tethering assay. We immunoprecipitated FLAG-tagged ERG from cells expressing the $R-L u c-8 M S 2$ transcript and tested copurified RNAs for the presence of $R-L u c-8 M S 2$ mRNA by RT-qPCR. We detected association between FLAG-ERG and the reporter mRNA only after coexpression of a hemagglutinin (HA)-tagged RBPMS construct fused to the MS2CP binding module (HA-RBPMS-MS2-CP), thus demonstrating that tethering of RBPMS onto target mRNA promoted co-recruitment of ERG (Fig. 3b). Consistently with the idea that endogenous RBPMS and ERG might be part of the same mRNP complexes, both proteins colocalized in PBs (white arrows in Fig. 3c). The observation that some PBs contained ERG but not RBPMS (red arrows in Fig. 3c) suggested that ERG might be recruited to mRNPs via RBPMS-independent mechanisms, most probably through interaction with other RBPs.

To support the idea that RBPMS might acquire the ability to recruit CNOT2 via its association with ERG, we assessed RBPMS-CNOT2 interaction in HEK-293 cells, which do not express detectable levels of endogenous ERG ${ }^{18}$. Myc-CNOT2 coimmunoprecipitated only with HA-RBPMS from cells ectopically expressing FLAG-ERG, thus indicating that the presence of ERG promoted association between RBPMS and CNOT2 (Fig. 3d). To formally demonstrate that ERG, RBPMS and CNOT2 can coexist within the same complex, we coexpressed FLAG-ERG, HA-RBPMS and Myc-CNOT2 in HEK-293 cells and immunoprecipitated ERG-associated complexes with anti-FLAG antibodies. After elution with a triple-FLAG $(3 \times$ FLAG $)$ peptide, we immunoprecipitated HA-RBPMS from the FLAG eluate and examined the presence of Myc-CNOT2 by western blotting. We specifically detected CNOT2 in the ERG-RBPMS immunoprecipitate, demonstrating that the three proteins coexist within the same complex 
a

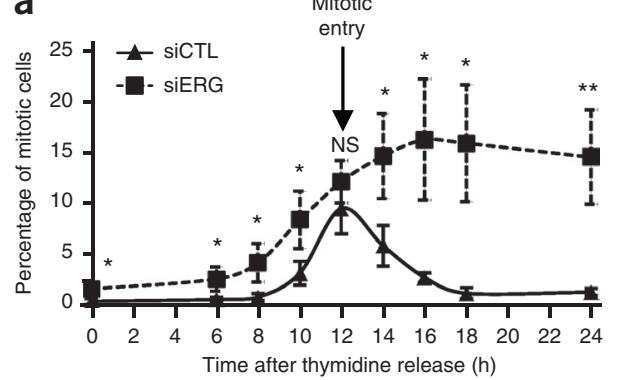

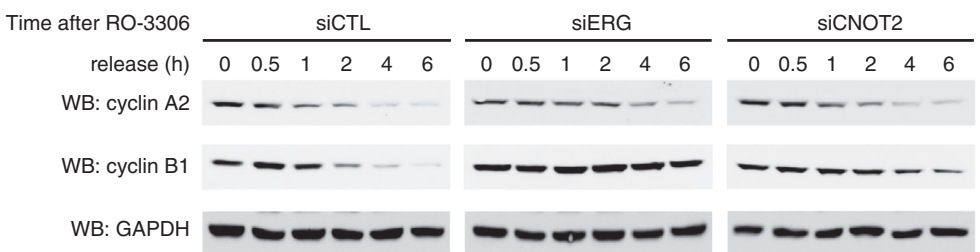

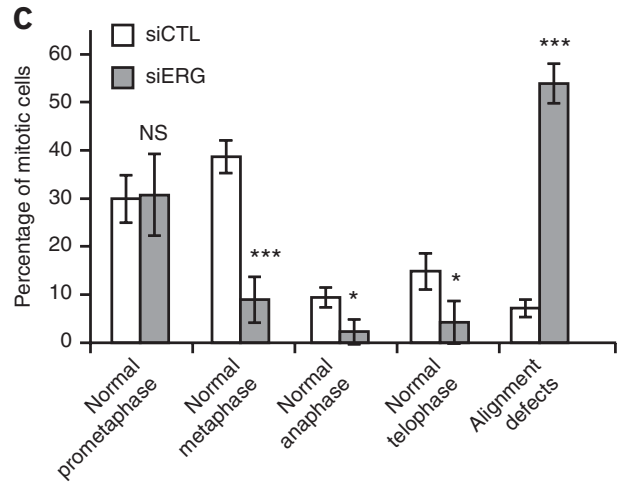

d

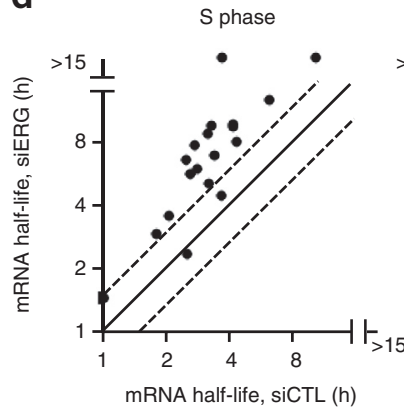

G2 phase

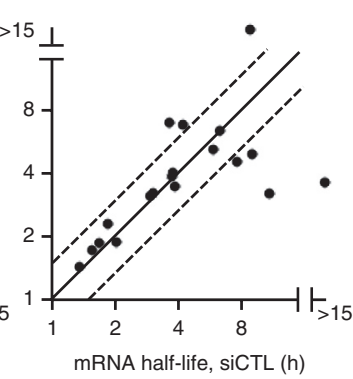

Mitosis

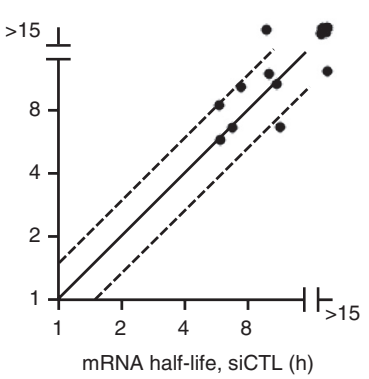

Figure 5 ERG depletion induces blockage in early metaphase. (a) FACS analysis using anti-phospho-histone H3 and propidium iodide labeling. Samples are from HeLa cells transfected with siCTL or siERG, synchronized in early S phase with a double thymidine block and then released. Results are shown as means \pm s.d. ( $n=3$ independent experiments). ${ }^{*} P<0.5 ;{ }^{*} P<0.01$; NS, not significant compared to siCTL by two-tailed unpaired Student's $t$ test. (b) Western blot against cyclin A2, cyclin B1 and GAPDH. Samples are lysates from HeLa cells transfected with siCTL, siERG or siCNOT2, synchronized at the G2-M boundary and released into mitosis. GAPDH is a loading control. Quantifications of western blots are shown in Supplementary Figure 5a. Uncropped blots are shown in Supplementary Data Set 1. (c) Quantification of cells in normal mitotic phases (additional data in Supplementary Fig. 5b) or showing mitotic defects (Fig. 6a,b). Samples are from HeLa cells transfected with siCTL or siERG. Results are shown as means \pm s.d. ( $n=3$ independent experiments with 50-100 mitotic cells counted in each replicate). ${ }^{*} P<0.05 ;{ }^{* * *} P<0.001 ; \mathrm{NS}$, not significant compared to siCTL by two-tailed unpaired Student's $t$ test. (d) RT-qPCR decay analysis of the 19 validated ERG mitotic targets (Fig. 4d and Supplementary Fig. 4b). Samples are RNA from HeLa cells transfected with either siCTL or siERG, synchronized in S phase, late G2 phase or mitosis, and treated with ActD for 0,2 or $4 \mathrm{~h}$. The representation is similar to that in Figure $4 \mathbf{d}(n=4,3$ and 3 independent experiments for $\mathrm{S}$, late $\mathrm{G} 2$ and mitotic cells, respectively). For clarity, s.d. are not shown but are displayed in Supplementary Figure 5c. Source data for a, c and d are available online.

(Fig. 3e). Consistently with this idea, we also found that endogenous RBPMS coimmunoprecipitated with CNOT2 and its partner CNOT3 (Supplementary Fig. 3e) and observed colocalization of ERG, RBPMS and CNOT2 in cytoplasmic granules of HeLa cells (Supplementary Fig. 3f, white arrows). Again, some ERG granules were negative for RBPMS, thus further suggesting that ERG might partner with other RBPs (Supplementary Fig. 3f, red arrows). Similarly, ERG and RBPMS also colocalized with the CNOT6 deadenylase subunit of CCR4-NOT. Altogether, our data provide compelling evidence that ERG relies on its interaction with RBPs such as RBPMS to associate with transcripts, whose degradation it promotes via recruitment of CNOT2.

ERG, RBPMS and CNOT2 promote degradation of mitotic mRNAs To identify cellular mRNAs targeted by ERG-mediated degradation, we analyzed genome-wide mRNA stability in ERG-depleted HeLa cells. We collected samples at $0,0.5,1,2$ and $4 \mathrm{~h}$ after transcription inhibition by ActD from cells transfected with siRNA against ERG (siERG) or control siRNA (siCTL) and assessed the decrease in the abundance of individual mRNAs over time. With this approach, we identified 196 mRNAs (corresponding to 186 individual genes) whose stability was most significantly increased in the absence of ERG (Fig. 4a, green dots, Supplementary Table 2 and http://www.etsinteractome.ulg.ac.be/degradation_erg/). Using our recent photoactivatable-ribonucleoside-enhanced cross-linking and immunoprecipitation (PAR-CLIP) data on RBPMS ${ }^{36}$, we found that these ERG degradation targets were strongly enriched in RBPMSassociated mRNAs, thus supporting a functional role for RBPMS in ERG-mediated decay (Fig. 4b). However, RBPMS-bound mRNAs were significantly more responsive than nontargets to stabilization after ERG knockdown (Fig. 4c). These findings further support our model in which ERG is recruited to specific mRNAs by various RBP partners, which include RBPMS.

Gene ontology (GO) analysis of ERG decay targets highlighted a significant enrichment in genes involved in cell-cycle regulation, including 22 mRNAs directly linked to mitosis (Supplementary Fig. 4a). Providing a high degree of confidence in the set of ERG mRNA targets, we verified the stabilization of 19 (85\%) of these 22 mitotic mRNAs by RT-qPCR (Fig. 4d, dots above the upper dashed line; details in Supplementary Fig. 4b). Interestingly, the silencing of CNOT2 and RBPMS increased the stability of 18 (95\%) and 11 (60\%), respectively, of the 19 verified endogenous ERG mitotic targets, thus providing further support to the idea that CNOT2 and RBPMS are key partners in ERG-mediated decay (Fig. 4d and Supplementary Fig. 4b,c).

Because our observations indicated that ERG might specifically target mitosis-related transcripts, we tested the importance of ERG-mediated mRNA decay in normal mitotic progression. Mitotic index analysis on synchronized cells showed that ERG-deficient cells progressed through $S$ phase and entered mitosis with kinetics similar to the kinetics of the control population, but they displayed a severe delay in the completion of mitosis (Fig. 5a). To refine these observations, we 
a

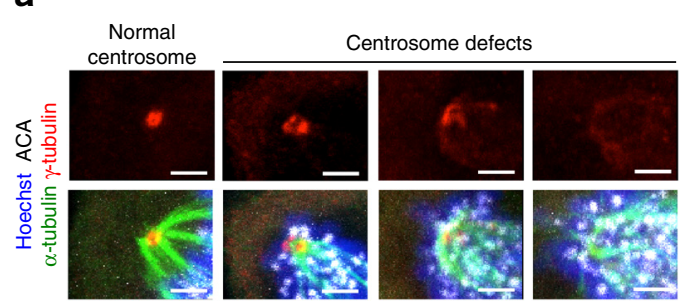

b

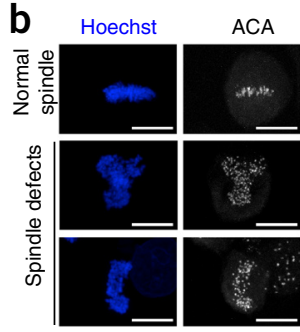

C

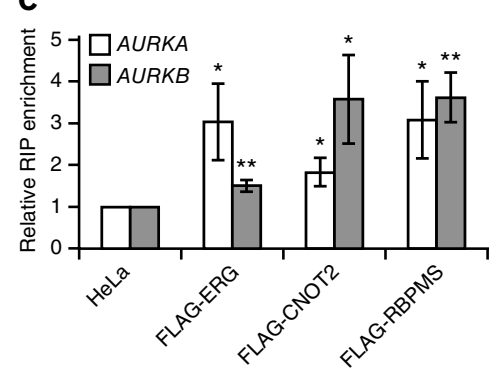

e Time after thymidine

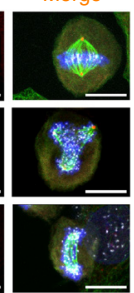

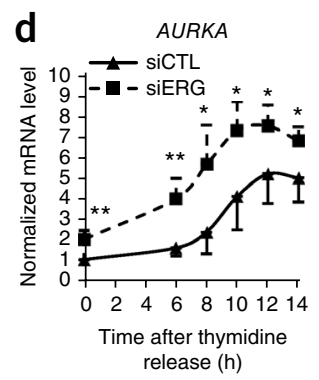

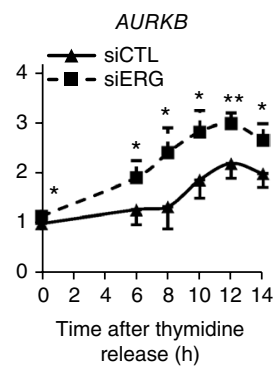

f

Figure 6 siERG-induced mitotic defects are due to overactivation of Aurora signaling. (a,b) Immunofluorescence of anticentromere antibodies (ACA) (centromeres, white), $\alpha$-tubulin (mitotic spindles, green), $\gamma$-tubulin (centrosomes, red) and Hoechst (chromosomes, blue) in HeLa cells transfected with anti-ERG siRNA (siERG). Scale bars, $2 \mu \mathrm{m}$ (a) and $10 \mu \mathrm{m}$ (b). Quantifications are shown in Supplementary Figure 6a-d. (c) Anti-FLAG immunoprecipitation and RT-qPCR analysis of AURKA and AURKB mRNAs. Samples are from HeLa cell lines expressing FLAG-tagged ERG, CNOT2 or RBPMS. Levels of $A U R K A$ and $A U R K B$ mRNAs in anti-FLAG immunoprecipitates are expressed as a percentage of the corresponding inputs, normalized to those of $18 \mathrm{~S}$ RNA and set to 1 in the control condition. Results are shown as means \pm s.d. ( $n=3$ independent experiments). ${ }^{*} P<0.05$; ${ }^{* *} P<0.01$ compared to control HeLa cells by two-tailed unpaired Student's $t$ test. (d) RT-qPCR analysis of the expression level of AURKA and AURKB mRNAs. Samples are from HeLa cells transfected with siCTL or siERG and released after an early S-phase double thymidine block. For standardization, mRNA levels in control cells at $t=0 \mathrm{~h}$ were set to 1 . Results are shown as means $\pm \mathrm{s}$.d. ( $n=4$ independent experiments). ${ }^{*} P<0.05$; ${ }^{* *} P<0.01$ compared to siCTL by two-tailed unpaired Student's $t$ test. (e) Western blot against AURKA, AURKB, phospho (p)-Aurora, cyclin E1 and GAPDH. Samples are from HeLa cells prepared as in d. Cyclin E1 was used to follow S-phase progression. GAPDH is a loading control. Quantifications of western blots are presented in Supplementary Figure 6i. Uncropped blots are shown in Supplementary Data Set 1. (f) Quantification of siERG-induced mitotic defects (in a,b). Samples are from HeLa cells transfected with either siCTL or siERG, and either left nontreated (NT) or treated with a suboptimal concentration of the Aurora inhibitor ZM447439 (ZM $0.5 \mu \mathrm{g} / \mathrm{ml})$. Results are individual percentages $\left(n=2\right.$ independent experiments, ). ${ }^{*} P<0.05,{ }^{* *} P<0.01$; NS, not significant compared to nontreated (NT) cells by two-tailed unpaired Student's $t$ test. Source data for $\mathbf{c}, \mathbf{d}$ and $\mathbf{f}$ are available online.

synchronized cells at the G2-M boundary and analyzed the levels of cyclins A2 and B1, whose sequential degradation during the prometaphase-metaphase and metaphase-anaphase transitions, respectively, are used to monitor progression through mitosis ${ }^{38}$. Both control and ERG-depleted cells proceeded through prometaphase and engaged normally in early metaphase, as indicated by the disappearance of cyclin A2 (Fig. 5b and Supplementary Fig. 5a). However, degradation of cyclin B1 was significantly retarded in siERG-treated cells, thus indicating that ERG depletion prevented progression through metaphase and mitosis completion. Further supporting this possibility, quantification of cells in the various mitotic phases showed that ERGknockdown cells did not proceed to normal metaphase (Fig. 5c and Supplementary Fig. 5b). Interestingly, depletion of CNOT2, which led to the stabilization of most of the mitotic ERG-target mRNAs (Fig. 4d), had a similar effect on mitotic progression, although this effect was less pronounced (Fig. 5b and Supplementary Fig. 5a). Unfortunately, RBPMS-depleted cells could not be tested in this assay, because G2-M blockage induced massive cell death in these cells.

On the basis of the assumption that bulk mRNA degradation would be turned off during mitosis ${ }^{39}$, we reasoned that the stabilization of mitotic mRNAs observed in ERG-deficient cells might be a consequence rather than a cause of the mitotic delay. To investigate this issue, we examined the decay of validated ERG mitotic targets in S, late G2 and early M phases of the cell cycle (Fig. 5d and Supplementary Fig. 5c). Whereas the absence of ERG had little to no significant effect on the mRNA turnover of its mitotic targets during G2 or M phases, it correlated with significant stabilization of the majority of the transcripts during $S$ phase. These analyses indicated that the stabilizing effect associated with ERG depletion mainly affected mRNA during $S$ phase, and it was thus unlikely that the longer time spent by ERG-depleted cells in mitosis accounted for the increase in mRNA stability.

ERG-mediated decay is critical for normal mitotic completion To determine how ERG depletion affected mitotic progression, we looked for potential mitotic defects in siERG-treated cells. Compared with control cells, cells lacking ERG often exhibited abnormal maturation and fragmentation of their centrosomes (Fig. 6a and Supplementary Fig. 6a). Loss of ERG was also associated with multipolar spindles and aberrant bipolar spindle structures (Fig. $6 \mathbf{b}$ and Supplementary Fig. $\mathbf{6 b}, \mathbf{c}$ ), which correlated with chromosome alignment defects (Fig. 6b and Supplementary Fig. 6d). Further strengthening the functional links among ERG, RBPMS and CNOT2, we observed similar mitotic abnormalities in RBPMS- and CNOT2depleted cells (Supplementary Fig. 6e).

Mitotic defects induced by ERG depletion are similar to those observed in cells overexpressing the Aurora A (AURKA) and B (AURKB) serine/threonine mitotic kinases ${ }^{40,41}$. Remarkably, mitotic ERG mRNA targets comprised transcripts encoding both AURKA and AURKB as well as multiple regulators (AUNIP and Borealin (CDCA8)), substrates (CDC25, Hice1 (HAUS8), CENPA, Borealin (CDCA8) and CDCA2) and interaction partners (PSRC1) of the Aurora kinases (Supplementary Fig. 4b). We thus speculated that the mitotic defects observed in ERG-deficient cells might result from 
Figure 7 ERG controls decay of Aurora-related mRNA transcripts in the cytoplasm via RBPMS and CNOT2. Model illustrating the new function of ERG in mRNA decay identified in this study. A portion of nuclear ERG might be recruited to the promoters of genes involved in the Aurora pathway. At some stage during or after transcription, ERG is loaded onto the transcripts via interaction of its CTAD with RBPMS and presumably other RNA-binding proteins (RBPs). At an undefined stage, ERG aids in recruiting the CCR4-NOT deadenylation complex, by virtue of its capacity to interact with CNOT2 via its ATAD. The ERG-RBPMS-CNOT2-mRNA complex accumulates in $\mathrm{P}$ bodies, where poly(A)-tail shortening and mRNA degradation are carried out. ERG-mediated degradation of Aurorarelated transcripts occurs specifically during $S$ phase and counteracts mRNA synthesis before entry into mitosis. The balance between mRNA synthesis and ERG-dependent decay tightly controls the levels of Aurorarelated transcripts and ensures proper regulation of Aurora signaling activity during mitosis. Pol, polymerase.

impaired degradation and accumulation of Aurora-related mRNAs, thus resulting in overactivation or untimely activation of Aurora signaling. In agreement with this possibility, depletion of ERG with two independent siRNAs reduced the S-phase-associated decay of Aurora-related mRNAs, including AURKA and AURKB (Fig. 5d and Supplementary Fig. 6f for siERG 1 and siERG 2, respectively). To refine these observations, we counted single AURKA transcripts in the cytoplasm and nuclei of siCTL- and siERG-transfected cells arrested in S phase, by using single-molecule RNA fluorescence in situ hybridization (RNA smFISH). Compared with control cells, ERG-deficient cells contained significantly more AURKA transcripts in their cytoplasm, thus supporting the idea of ERG being important for the cytoplasmic decay of AURKA mRNA (Supplementary Fig. 6g,h). To strengthen these findings, we tested whether ERG associates with $A U R K A$ and $A U R K B$ mRNAs. By performing RIP on extracts from inducible HeLa cells expressing FLAG-ERG close to endogenous levels, and subsequent RT-qPCR analysis, we found that ERG immunoprecipitates were significantly enriched in AURKA and $A U R K B$ mRNAs (Fig. 6c). RBPMS and CNOT2 also associated with $A U R K A$ and $A U R K B$ mRNAs (Fig. 6c), thus supporting the possibility that these proteins participate in ERG-mediated mRNA decay.

Consistently with a defect in ERG-mediated decay during $\mathrm{S}$ phase, ERG-deficient cells accumulated higher levels of AURKA and AURKB mRNAs as they progressed through $S$ phase and consequently exhibited more transcripts in G2 and when entering mitosis (Fig. 6d). The higher levels of $A U R K A$ and $A U R K B$ mRNAs in ERG-depleted cells translated into increased expression of Aurora kinases (Fig. 6e and Supplementary Fig. 6i). Consequently, cells lacking ERG activated Aurora kinases prematurely and thus entered mitosis with hyperactivated Aurora signaling, as compared with control cells (Fig. 6e and Supplementary Fig. 6j). Suboptimal concentrations of ZM447439 $(0.5 \mu \mathrm{g} / \mathrm{ml})$, a specific inhibitor of Aurora A and B, significantly reverted the various mitotic defects associated with a lack of ERG, without substantially affecting the control cells (Fig. 6f). This result demonstrated the causal role of Aurora overactivation in siERG-associated mitotic defects.

Collectively, these results clearly establish a role of ERG in mRNA decay and reveal an important biological context for this new function by showing that ERG-mediated degradation of Aurora-related transcripts is critical to ensure proper progression through metaphase during mitosis (Fig. 7).

\section{DISCUSSION}

In terms of efficiency, robustness, adaptability and precision of the gene-expression response, the advantages of coupling mRNA synthesis to degradation are obvious. However, mostly because of the physical barrier of the nuclear envelope between the two

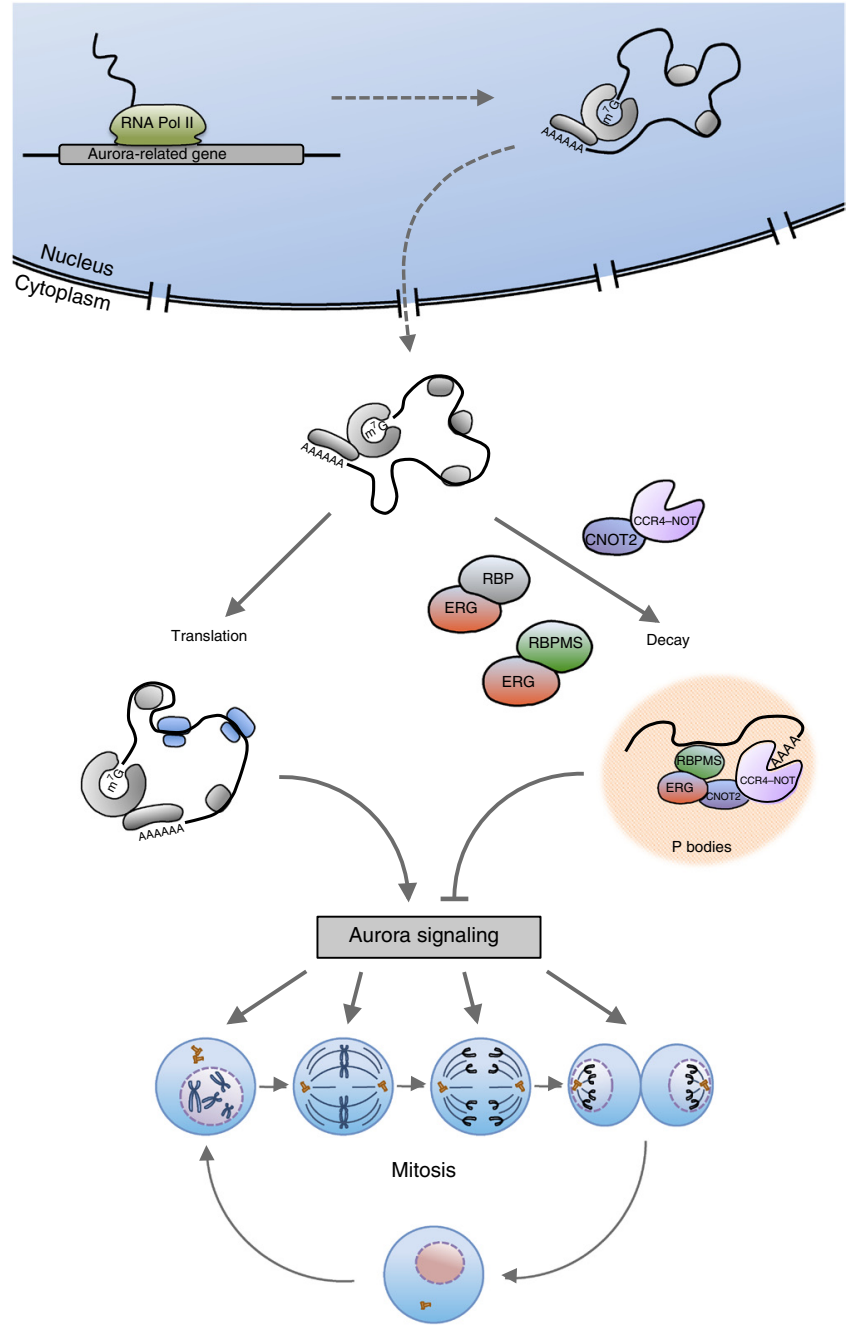

processes, the idea of a possible connection between transcription and decay has long been set aside.

In this work, we provide a substantial body of evidence demonstrating that the transcription factor ERG can achieve control of gene expression by directly affecting cytoplasmic mRNA decay, the endpoint of mRNA lifespan. Although the present demonstration that mammalian TFs can actively participate in the process of cytoplasmic mRNA degradation was highly unexpected, our work comes in the wake of recent genetic and molecular evidence establishing functional coupling between mRNA synthesis and degradation in yeast ${ }^{42-44}$. In particular, specific promoter sequences in yeast have been shown to influence cytoplasmic mRNA decay, presumably through their associated $\mathrm{TFs}^{12,13}$.

Erg proteins are canonical mammalian TFs that bind to specific recognition elements (Ets-binding sites) in the promoters of their transcriptional targets. Using available ERG chromatin immunoprecipitation (ChIP) data sets ${ }^{45}, 46$, we found that ERG-target mRNAs are statistically enriched in ERG-associated genes $\left(P=5.4310^{-13}\right.$ by Pearson's chi-squared test; data not shown). Similarly, mRNAs synthesized from ERG-associated genes were significantly more stabilized than nontargets after ERG knockdown $\left(P=1.0810^{-9}\right.$ by KolmogorovSmirnov test; data not shown). These results indicate a strong correlation between the presence of ERG at a specific gene locus and its ability to control the degradation of the corresponding mRNA. In yeast, the emerging model suggests that the TFs may control mRNA fate by coordinating the loading of mRNA-decay regulators, 
such as components of deadenylation machineries on the transcribing mRNA (so-called mRNA imprinting ${ }^{14}$ ). In this model, the coordinating $\mathrm{TF}$ is not incorporated into the mRNP. In contrast, our observations suggest that ERG is indeed an mRNP component and that it actively participates in the degradation of the imprinted mRNA in the cytoplasm (Fig. 7). It will now be essential to determine whether the ERG molecules that are bound to promoter sequences and control transcriptional events are also the ones that are incorporated into mRNPs and are involved in the regulation of mRNA decay.

Our $\mathrm{Y} 2 \mathrm{H}$ screening revealed that ERG interacts with at least two RBPs implicated in mRNA decay and splicing. Although we focused our attention on mRNA decay, our results suggest that ERG proteins might achieve control of gene expression by orchestrating multiple processes between transcription and decay. In addition, we have recently found 15 TFs among the RBPMS partners in our proteome-scale human interactome (GO: sequence-specific DNA binding) ${ }^{47}$. Therefore, a wide range of TFs and RBPs may actually collaborate in coupling transcriptional and post-transcriptional processes and machineries through the formation of highly versatile functional platforms. In terms of RNA degradation, targeting of the CCR4-NOT complex to specific mRNAs is known to involve interactions with sequence-specific RBPs ${ }^{3}$. On the basis of our study, we speculate that an additional level of specificity might be achieved through interactions with sequence-specific DNAbinding factors, such as ERG. In this case, transcript-specific targeting of CNOT2 would rely combinatorially on its promoter-specific recruitment by TFs and RBP-dependent loading onto the transcribing mRNA. It is also notable that the coordinated functions of CCR4-NOT in transcription and mRNA decay rely more specifically on its NOT2-NOT3 module, which interacts with ERG ${ }^{11}$.

The levels and activities of Aurora kinases are precisely modulated throughout the cell cycle via a complex network of regulatory mechanisms that involves control of mRNA synthesis and translation rates, post-translational modifications, association with specific cofactors and protein degradation ${ }^{48}$. Surprisingly, the contribution of mRNA decay has been largely overlooked in studies of mitosis regulation $^{13,49,50}$. Here, we demonstrate that mRNA degradation of Aurorarelated transcripts prominently contributes to mitotic progression in higher eukaryotes. Our findings provide a new model of mitosis regulation in which mRNA decay participates in the orchestration of sequential mitotic events by ensuring the rapid and robust inactivation of critical mitotic effectors.

Overall, our study defines a new and unexpected function of the transcription factor ERG in cytoplasmic mRNA decay. The possibility that our findings might be extended to a wide range of transcription factors not only affects the current model of eukaryotic gene regulation but also provides a mechanistic model for how gene-specific and condition-specific coupling between mRNA synthesis and degradation might be achieved ${ }^{51,52}$.

\section{METHODS}

Methods and any associated references are available in the online version of the paper.

Accession codes. Microarray data have been deposited in the Gene Expression Omnibus database under accession code GSE80395.

Note: Any Supplementary Information and Source Data files are available in the online version of the paper.

\section{ACKNOWLEDGMENTS}

We thank the Vidal laboratory at the Center for Cancer Systems Biology at the Dana-Farber Cancer Institute (CCSB, DFCI) for supplying reagents, advice and technical support for the HT-Y2H screen. We thank the Tuschl laboratory (Howard Hughes Medical Institute, Rockefeller University) for sharing reagents. We are grateful to E. Izaurralde (European Molecular Biology Laboratory) for kindly providing HA-tagged CNOT2, CNOT3, CNOT6 and CNOT. We would like to thank the laboratories of the Molecular Biology in Diseases Unit (GIGAR, ULg) for helpful discussions and C. Vindry from the Kruys laboratory for technical assistance. We thank the GIGA interactomics, imaging and flow cytometry and genotranscriptomics platforms for their technical support. This work was supported by grants from the University of Liège, the Fonds Léon Frédéricq, the Belgian Foundation against Cancer (Special Equipment Fund), the Belgian National Fund for Scientific Research (FNRS), Télévie, the Interuniversity Attraction Poles Program-Belgian Science Policy (IUAP-BELSPO PVI/28 and PVII/13 to F.D. and M. Bollen) and the National Human Genome research Institute (grant R01HG001715 to M.V. and D.E.H.). X.R., J-C.T. and N.S. are supported as an FNRS Research Fellow, Research Associate and Postdoctoral Fellow, respectively. C.D. and K.G. are supported as Télévie-FNRS PhD students.

\section{AUTHOR CONTRIBUTIONS}

F.D. and X.R. conceived the project and designed the experiments.

X.R. performed most experiments and processed and analyzed the data.

C.D., J.B., E.M., M.C., P.D., M.L., R.S., K.G., R.K., I.S. and V.K. assisted with experiments. D.E.H., M.V., N.S. and J.-C.T. helped perform and analyze the HT-Y2H screen. S.B. performed bioinformatic analysis for the transcriptome-wide decay experiments and contributed to the corresponding manuscript sections. M. Bollen, M. Beullens and B.L. contributed to analysis of ERG in mitotic progression and Aurora kinase regulation and participated in writing the corresponding manuscript sections. T.A.F. assisted in analyzing RBPMS CLIP data. F.D. and X.R. wrote the manuscript, which was edited by all authors.

\section{COMPETING FINANCIAL INTERESTS}

The authors declare no competing financial interests.

Reprints and permissions information is available online at http://www.nature.com/ reprints/index.html.

1. Braun, K.A. \& Young, E.T. Coupling mRNA synthesis and decay. Mol. Cell. Biol. 34, 4078-4087 (2014).

2. Darnell, J.E. Jr. Reflections on the history of pre-mRNA processing and highlights of current knowledge: a unified picture. RNA 19, 443-460 (2013).

3. Wahle, E. \& Winkler, G.S. RNA decay machines: deadenylation by the Ccr4-not and Pan2-Pan3 complexes. Biochim. Biophys. Acta 1829, 561-570 (2013).

4. Keene, J.D. RNA regulons: coordination of post-transcriptional events. Nat. Rev. Genet. 8, 533-543 (2007).

5. Chen, C.Y. \& Shyu, A.B. Deadenylation and P-bodies. Adv. Exp. Med. Biol. 768, 183-195 (2013).

6. Marguerat, S., Lawler, K., Brazma, A. \& Bähler, J. Contributions of transcription and mRNA decay to gene expression dynamics of fission yeast in response to oxidative stress. RNA Biol. 11, 702-714 (2014).

7. Shalem, O., Groisman, B., Choder, M., Dahan, O. \& Pilpel, Y. Transcriptome kinetics is governed by a genome-wide coupling of mRNA production and degradation: a role for RNA Pol II. PLoS Genet. 7, e1002273 (2011).

8. Sun, M. et al. Global analysis of eukaryotic mRNA degradation reveals Xrn1dependent buffering of transcript levels. Mol. Cell 52, 52-62 (2013).

9. Sun, M. et al. Comparative dynamic transcriptome analysis (cDTA) reveals mutual feedback between mRNA synthesis and degradation. Genome Res. 22, 1350-1359 (2012).

10. Collart, M.A. \& Timmers, H.T. The eukaryotic Ccr4-not complex: a regulatory platform integrating mRNA metabolism with cellular signaling pathways? Prog. Nucleic Acid Res. Mol. Biol. 77, 289-322 (2004).

11. Miller, J.E. \& Reese, J.C. Ccr4-Not complex: the control freak of eukaryotic cells Crit. Rev. Biochem. Mol. Biol. 47, 315-333 (2012).

12. Bregman, A. et al. Promoter elements regulate cytoplasmic mRNA decay. Cell 147 1473-1483 (2011).

13. Trcek, T., Larson, D.R., Moldón, A., Query, C.C. \& Singer, R.H. Single-molecule mRNA decay measurements reveal promoter- regulated mRNA stability in yeast. Cell 147, 1484-1497 (2011).

14. Haimovich, G., Choder, M., Singer, R.H. \& Trcek, T. The fate of the messenger is pre-determined: a new model for regulation of gene expression. Biochim. Biophys. Acta 1829, 643-653 (2013).

15. Sharrocks, A.D. The ETS-domain transcription factor family. Nat. Rev. Mol. Cell Biol. 2, 827-837 (2001).

16. Hollenhorst, P.C., Shah, A.A., Hopkins, C. \& Graves, B.J. Genome-wide analyses reveal properties of redundant and specific promoter occupancy within the ETS gene family. Genes Dev. 21, 1882-1894 (2007).

17. Hollenhorst, P.C., McIntosh, L.P. \& Graves, B.J. Genomic and biochemical insights into the specificity of ETS transcription factors. Annu. Rev. Biochem. 80, 437-471 (2011).

18. Uhlen, M. et al. Towards a knowledge-based Human Protein Atlas. Nat. Biotechnol. 28, 1248-1250 (2010) 
19. Eulalio, A., Behm-Ansmant, I. \& Izaurralde, E. P bodies: at the crossroads of posttranscriptional pathways. Nat. Rev. Mol. Cell Biol. 8, 9-22 (2007).

20. Castello, A. et al. Insights into RNA biology from an atlas of mammalian mRNAbinding proteins. Cell 149, 1393-1406 (2012).

21. Singh, G., Ricci, E.P. \& Moore, M.J. RIPiT-Seq: a high-throughput approach for footprinting RNA:protein complexes. Methods 65, 320-332 (2014).

22. Norris, J.D., Fan, D., Sherk, A. \& McDonnell, D.P. A negative coregulator for the human ER. Mol. Endocrinol. 16, 459-468 (2002).

23. Decker, C.J. \& Parker, R. P-bodies and stress granules: possible roles in the control of translation and mRNA degradation. Cold Spring Harb. Perspect. Biol. 4, a012286 (2012).

24. Tourrière, $\mathrm{H}$. et al. The RasGAP-associated endoribonuclease G3BP assembles stress granules. J. Cell Biol. 160, 823-831 (2003).

25. Kedersha, N.L., Gupta, M., Li, W., Miller, I. \& Anderson, P. RNA-binding proteins TIA-1 and TIAR link the phosphorylation of elF-2 alpha to the assembly of mammalian stress granules. J. Cell Biol. 147, 1431-1442 (1999).

26. Barreau, C., Watrin, T., Beverley Osborne, H. \& Paillard, L. Protein expression is increased by a class III AU-rich element and tethered CUG-BP1. Biochem. Biophys. Res. Commun. 347, 723-730 (2006).

27. Hoesel, B. et al. Sequence-function correlations and dynamics of ERG isoforms: ERG8 is the black sheep of the family. Biochim. Biophys. Acta 1863, 205-218 (2016).

28. Liu, J. et al. A role for the P-body component GW182 in microRNA function. Nat. Cell Biol. 7, 1261-1266 (2005).

29. Dreze, M. et al. High-quality binary interactome mapping. Methods Enzymol. 470 , 281-315 (2010)

30. Ito, K. et al. CNOT2 depletion disrupts and inhibits the CCR4-NOT deadenylase complex and induces apoptotic cell death. Genes Cells 16, 368-379 (2011).

31. Winkler, G.S., Mulder, K.W., Bardwell, V.J., Kalkhoven, E. \& Timmers, H.T. Human Ccr4-Not complex is a ligand-dependent repressor of nuclear receptor-mediated transcription. EMBO J. 25, 3089-3099 (2006).

32. Boland, A. et al. Structure and assembly of the NOT module of the human CCR4-NOT complex. Nat. Struct. Mol. Biol. 20, 1289-1297 (2013).

33. Barckmann, B. \& Simonelig, M. Control of maternal mRNA stability in germ cells and early embryos. Biochim. Biophys. Acta 1829, 714-724 (2013).

34. Kuroyanagi, H. Fox-1 family of RNA-binding proteins. Cell. Mol. Life Sci. 66, 3895-3907 (2009).

35. Song, H.W. et al. Hermes RNA-binding protein targets RNAs-encoding proteins involved in meiotic maturation, early cleavage, and germline development. Differentiation 75, 519-528 (2007).

36. Farazi, T.A. et al. Identification of the RNA recognition element of the RBPMS family of RNA-binding proteins and their transcriptome-wide mRNA targets. RNA 20, 1090-1102 (2014).
37. Sun, S., Zhang, Z., Fregoso, O. \& Krainer, A.R. Mechanisms of activation and repression by the alternative splicing factors RBFOX1/2. RNA 18, 274-283 (2012).

38. Pines, J. \& Hunter, T. Human cyclins A and B1 are differentially located in the cell and undergo cell cycle-dependent nuclear transport. J. Cell Biol. 115, 1-17 (1991).

39. Ross, J. A hypothesis to explain why translation inhibitors stabilize mRNAs in mammalian cells: mRNA stability and mitosis. BioEssays 19, 527-529 (1997).

40. Nikonova, A.S., Astsaturov, I., Serebriiskii, I.G., Dunbrack, R.L. Jr. \& Golemis, E.A. Aurora A kinase (AURKA) in normal and pathological cell division. Cell. Mol. Life Sci. 70, 661-687 (2013).

41. Ruchaud, S., Carmena, M. \& Earnshaw, W.C. Chromosomal passengers: conducting cell division. Nat. Rev. Mol. Cell Biol. 8, 798-812 (2007).

42. Goler-Baron, V. et al. Transcription in the nucleus and mRNA decay in the cytoplasm are coupled processes. Genes Dev. 22, 2022-2027 (2008).

43. Lotan, R. et al. The RNA polymerase II subunit Rpb4p mediates decay of a specific class of mRNAs. Genes Dev. 19, 3004-3016 (2005).

44. Lotan, R., Goler-Baron, V., Duek, L., Haimovich, G. \& Choder, M. The Rpb7p subunit of yeast RNA polymerase II plays roles in the two major cytoplasmic mRNA decay mechanisms. J. Cell Biol. 178, 1133-1143 (2007).

45. Yu, J. et al. An integrated network of androgen receptor, polycomb, and TMPRSS2 ERG gene fusions in prostate cancer progression. Cancer Cell 17, 443-454 (2010).

46. Wilson, N.K. et al. Combinatorial transcriptional control in blood stem/progenitor cells: genome-wide analysis of ten major transcriptional regulators. Cell Stem Cell 7, 532-544 (2010).

47. Rolland, $\mathrm{T}$. et al. A proteome-scale map of the human interactome network. Cell 159, 1212-1226 (2014).

48. Carmena, M., Ruchaud, S. \& Earnshaw, W.C. Making the Auroras glow: regulation of Aurora A and B kinase function by interacting proteins. Curr. Opin. Cell Biol. 21, 796-805 (2009).

49. Gill, T., Cai, T., Aulds, J., Wierzbicki, S. \& Schmitt, M.E. RNase MRP cleaves the CLB2 mRNA to promote cell cycle progression: novel method of mRNA degradation. Mol. Cell. Biol. 24, 945-953 (2004).

50. Alvarez, B., Blanco, M.A. \& Moreno, S. The fission yeast APC activator Ste9 is regulated by mRNA decay. Cell Cycle 5, 865-868 (2006).

51. Pai, A.A. et al. The contribution of RNA decay quantitative trait loci to interindividual variation in steady-state gene expression levels. PLoS Genet. 8, e1003000 (2012).

52. Rabani, M. et al. Metabolic labeling of RNA uncovers principles of RNA production and degradation dynamics in mammalian cells. Nat. Biotechnol. 29, 436-442 (2011). 


\section{ONLINE METHODS}

Plasmids and antibodies. ORFs encoding FLI1, ERG, CNOT2 and RBPMS were obtained from the human ORFeome v5.1 (CCSB, Dana-Farber Cancer Institute) in pDONR223. The ORF encoding FEV was purchased from OriGene (pCMV6XL5-FEV, cat. no. SC126133). Full-length FEV, as well as all isolated domains of Erg members and deletion mutants of ERG, CNOT2 and RBPMS, were inserted into pDONR223 by Gateway recombination methodology (Invitrogen) with specific primers $5^{\prime}$ flanked by the following AttB1.1 and AttB2.1 Gateway sites: 5'-GGGGACAACTTTGTACAAAAAAGTTGGC(ATG)-3' (AttB1) and 5'-GG GGACAACTTTGTACAAGAAAGTTGA-3' (AttB2). Primer sequences are available upon request.

Inserts from pDONR223 were transferred by LR cloning (Invitrogen) into the different destination vectors: $\mathrm{Y} 2 \mathrm{H}$ expression vectors $\mathrm{pAD}$-dest-CYH (Gal4-AD $\mathrm{N}$-terminal tag), pDB-dest (Gal4-DB N-terminal tag) ${ }^{29}$, pDEST491 (YFP N-terminal tag), pDEST1899 (FLAG N-terminal tag), pCS3MTdest (Myc N-terminal tag, Addgene), pDEST475 (HA N-terminal tag, Invitrogen), pDEST1899-MS2-CP (FLAG-MS2-CP N-terminal tag) or pLenti CMVtight Blast DEST (Advanced TetOn system, Addgene). pDEST1899-MS2-CP was built by inserting the MS2-CP tag obtained from the pN-MS2-CP plasmid ${ }^{53}$ between the $3 \times$ FLAG sequence and the AttR1 recombination sequence of pDEST1899. The RFP-DCP1A construct was described previously ${ }^{54}$. All constructs were verified by sequencing. Details for the antibodies used in this study can be found in Supplementary Note 1.

Cell culture, transfection and treatments. HeLa, HEK-293, U2OS and MRC5 cells were purchased from ATCC. We generated HA-FLAG-tagged ERG, RBPMS and CNOT2-inducible stable HeLa cell lines as follows: HeLa cells were transduced with pLenti CMV rtTA3 Hygro (Addgene) (10 MOI) and selected with $100 \mu \mathrm{g} / \mathrm{ml}$ hygromycin B Gold (InvivoGen); rtTA3 HeLa cells were subsequently transduced with pLenti CMVtight Blast bearing sequences encoding HA-FLAGtagged RBPMS, ERG or CNOT2 (50 MOI) and were selected with $10 \mu \mathrm{g} / \mathrm{ml}$ blasticidin (InvivoGen). HeLa, HEK-293 and U2OS cell lines were maintained in 10\% FBS-complemented DMEM (Gibco) in standard cell-culture conditions. MRC5 cells were maintained in 10\% FBS-complemented EMEM (Gibco). All cell lines were verified for mycoplasma contamination on a monthly basis (Mycoalert Mycoplasma Detection Kit, Lonza).

DNA and siRNA were transfected into HeLa, U2OS and MRC5 cells with JetPrime (Polyplus transfection). For DNA-siRNA transfection, the medium of cells that had reached $80-50 \%$ confluency was exchanged with supplemented DMEM-OPTI-MEM(I) before transfection and with DMEM 12-24 h after transfection. DNA was transfected in HEK-293 cells through a classical calcium phosphate procedure. Cells were processed at $36 \mathrm{~h}$ or 60-72 $\mathrm{h}$ after DNA or siRNA transfection, respectively. The following siRNA duplexes were purchased from Eurogentec (Belgium): siERG 1, 5'-CGACA UCCUUCUCUCACAUtt- $3^{\prime}$; siERG 2, an equimolar pool of $5^{\prime}$-GUUGAUA AAGCCUUACAAAtt- $3^{\prime}$ and 5' ${ }^{\prime}$-UGCGUUUGUCAGAAUGAAGtt- $3^{\prime}$; siGW182, 5'-GUCGCAAAAUGGAGAUUGAtt- $3^{\prime}$; siCNOT2, 5'-CAUCUGAG UACUUAACGAAtt-3'; and control siRNA (SR-CL000-005). siRBPMS was an equimolar pool of two siRNAs from Santa Cruz (sc-77460).

For SG induction, HeLa cells were treated with $1 \mathrm{mM}$ arsenite for $30 \mathrm{~min}$ (to induce oxidative stress) or $600 \mathrm{mM}$ sorbitol for $1 \mathrm{~h}$ (to induce osmotic stress) or were heat-shocked at $46^{\circ} \mathrm{C}$ for $1 \mathrm{~h}$. Protein synthesis was blocked with $100 \mu \mathrm{g} / \mathrm{ml}$ cycloheximide for $1 \mathrm{~h}$. For mRNA decay experiments, transcription was blocked with $5 \mu \mathrm{M}$ ActD for up to $4 \mathrm{~h}$. Cells were treated with the Aurora kinase A and B inhibitor ZM447439 (Selleckchem) at the suboptimal concentration of $0.5 \mu \mathrm{g} / \mathrm{ml}$ for $48 \mathrm{~h}$ (medium was replaced after $24 \mathrm{~h}$ ).

Cells were blocked in S phase with a classical double-thymidine-block procedure with $2 \mathrm{mM}$ thymidine. When required, cells were transfected with siRNA at the first thymidine treatment. To block cells in G2 or mitosis, cells were blocked in $\mathrm{S}$ phase by a single thymidine block, released in culture medium for $3 \mathrm{~h}$ and treated for $12 \mathrm{~h}$ with a CDK1 inhibitor (RO-3306, $10 \mu \mathrm{M}$, Sigma-Aldrich) or nocodazole $(100 \mathrm{ng} / \mathrm{ml})$, respectively. When required, cells were siRNA transfected $18-24 \mathrm{~h}$ before the thymidine block.

RNA extraction and RT-qPCR. For expression studies and mRNA decay analyses, total RNA was extracted from cell pellets with a NucleoSpin RNA kit (Macherey-Nagel). For RNA-immunoprecipitations, total RNA was extracted from inputs or beads with TriPure Isolation Reagent (Roche). Total RNA was then reverse transcribed with random primers (Thermo Scientific), per the manufacturer's instructions. RT-qPCR reactions were performed with SYBR Green detection (Roche) and analyzed in triplicate on a LightCycler 480 (Roche). Sequences of RT-qPCR primers are shown in Supplementary Note 2.

In expression studies, relative target mRNA levels were calculated with the $\triangle \triangle \mathrm{Ct}$ method with GAPDH as an internal control. For mRNA decay analyses, signals were normalized to the average levels of three highly stable mRNAs (GAPDH, RPL32 and NDUFA12), and half-lives were calculated on the basis of first-order degradation kinetics. Normalizations of RIP experiments are indicated in the related paragraph.

Tethering degradation assay. Luciferase tethering assays were performed with HeLa cells cotransfected with control MS2-CP or various MS2-CP-tagged constructs, together with a bidirectional reporter encoding a control Firefly luciferase (F-Luc) and a targeted Renilla luciferase carrying or lacking eight repeats of the binding sequence for the MS2 coating peptide in its $3^{\prime}$ UTR (R-Luc-8MS2 and R-Luc-0MS2, respectively) ${ }^{26}$. For measuring luciferase activities, cell lysis and luciferase assays were performed in triplicate on a Lumat LB 9507 luminometer (EG\&G Berthold) with the Dual-Luciferase system (Promega). Importantly, for mRNA decay analyses, forward and reverse R-Luc primers were designed to anneal at two different exons to ensure specific amplification of spliced mRNA and to prevent any contamination from the transfected coding plasmid. The net effect of an MS2-CP-tagged protein on the degradation of the tethered $R$-Luc-8MS2 mRNA reporter was calculated as follows. For each tested protein, the ratio of R-Luc-8MS2 to R-Luc-0MS2 was calculated and normalized to the corresponding F-Luc ratio. The R-Luc/F-Luc ratios were then calculated and normalized to the control MS2-CP condition independently for each cell line or siRNA tested.

Immunofluorescence and confocal microscopy. HeLa cells were grown on glass slides, washed in warm PBS and fixed in warm $4 \%$ paraformaldehyde (PAF) for 15 min. Cells were then washed in PBS, permeabilized in PBS with $0.5 \%$ Triton X-100 for 5-10 min and incubated in blocking solution (PBS with $0.025 \%$ Tween20 and $10 \%$ FBS) for $30 \mathrm{~min}$. Staining was performed in optimized dilutions of primary antibody ( $2 \mathrm{~h}$ ) and Alexa-conjugated secondary antibody (30 min) in blocking solution. Cells were stained with Hoechst (1:50,000 in deionized water), washed three times for $5 \mathrm{~min}$ each in deionized water and mounted on glass coverslips with Mowiol (Sigma-Aldrich). For $\gamma$-tubulin staining, cells were permeabilized $5 \mathrm{~min}$ in cold methanol.

The smFISH protocol was derived from ref. 55. PAF-fixed cells were preferentially permeabilized with cold ethanol $\left(2 \mathrm{~h}\right.$ to overnight at $\left.4{ }^{\circ} \mathrm{C}\right)$, washed twice with smFISH wash buffer ( $4 \times$ SSC with $15 \%$ formamide) and subjected to hybridization $(4 \times$ SSC, $15 \%$ formamide, $10 \%$ dextran sulfate, $1 \mathrm{mg} / \mathrm{ml}$ yeast or Escherichia coli tRNA, $0.02 \%$ BSA and $125 \mathrm{mM}$ Stellaris RNA FISH probe cocktail (Biosearch Technologies)) for $3 \mathrm{~h}$ at $37^{\circ} \mathrm{C}$ in a humidified chamber. The RNA FISH probe cocktail was composed of 48 DNA probes ( $22 \mathrm{nt}$ long, TAMRA labeled), designed with an online tool (Stellaris, Biosearch Technologies) against the sequence of the full-length AURKA mRNA, which is conserved among all known isoforms. Cells were then stained with Hoechst and rinsed three times with smFISH wash buffer before mounting.

Slides were analyzed by confocal microscopy with an Olympus FluoView FV1000 confocal microscope with a $60 \times$ oil objective. Images were taken at $1024 \times$ 1024 pixel resolution and processed and assembled with FluoView viewer. smFISH labeling was subject to fading and needed to be rapidly analyzed; imaging required a Kalman correction to eliminate background ( $n=3$ images).

$\mathrm{PB}$ and SG counting was performed on cells reconstituted in 3D with IMARIS software. Surfaces were constructed from DCP1A (for PBs) or G3BP (for SGs) labeling, considering fluorescence signals above a control threshold, and were filtered for a minimum volume of 0.05 or $0.2 \mu \mathrm{m}^{3}$, respectively. Granules were counted with the appropriate IMARIS software function. Cell number was determined on the basis of the Hoechst signal, and the mean number of PBs or SGs per cell was calculated. The length of the mitotic spindle was calculated from individual cells analyzed in IMARIS. Single mRNA molecules (smFISH) were counted with the ad hoc ready-to-use StarSearch application (http://rajlab.seas. upenn.edu/), with a threshold of 40 . 
Oligo(dT) pulldowns. The oligo(dT) pulldown procedure was derived from refs. 20,21. HeLa cells were washed twice in cold PBS and either UV cross-linked $\left(0.4 \mathrm{~J} / \mathrm{cm}^{2}\right.$ of $365-\mathrm{nm}$ UV light with a Stratalinker 2400$)$ or paraformaldehyde fixed (scraped cells were resuspended in PBS with $0.1 \%$ paraformaldehyde and rotated for $10 \mathrm{~min}$ at room temperature, $10 \mathrm{ml}$ per $15-\mathrm{cm}$ dish). PAF fixation was quenched by glycine (final concentration of $125 \mathrm{mM}$ for $10 \mathrm{~min}$ at room temperature, with rotation), and cells were washed two times with cold PBS. UV-cross-linked and PAF-fixed cells were lysed in oligo(dT) lysis buffer (20 mM Tris-HCl, pH 7.5, $500 \mathrm{mM} \mathrm{LiCl}$, 0.5\% SDS, 1 mM EDTA, 1 mM DTT, cOmplete Protease Inhibitor (Roche), Halt Phosphatase Inhibitors (Thermo Scientific) and Protector RNase Inhibitor (Roche)), and cell lysates were homogenized by three or four passages through a syringe with a 0.4 -mm diameter needle (overhomogenization leads to less viscous lysates but also to mRNP breakage). Homogenized cell lysates were then incubated at $37^{\circ} \mathrm{C}$ for $30 \mathrm{~min}$ with or without RNase A ( $200 \mu \mathrm{g} / \mathrm{ml}$ Thermo Scientific) (neither DTT nor RNase protector was added in the RNase-treated samples used as negative controls for oligo(dT) pulldown). $1 \%$ of each cell lysate was kept for input, and the rest was incubated with oligo(dT) magnetic beads (New England BioLabs) for $2 \mathrm{~h}$ at $4{ }^{\circ} \mathrm{C}(25 \mu \mathrm{l}$ of beads per $15-\mathrm{cm}$ culture dish). Pulldowns were washed once with wash buffer I ( $20 \mathrm{mM}$ Tris-HCl, pH 7.5, $500 \mathrm{mM} \mathrm{LiCl}, 0.1 \%$ SDS, 1 mM EDTA and $1 \mathrm{mM}$ DTT), once with wash buffer II (20 mM Tris- $\mathrm{HCl}, \mathrm{pH} 7.5,500 \mathrm{mM} \mathrm{LiCl}$, $1 \mathrm{mM}$ EDTA and $1 \mathrm{mM} \mathrm{DTT}$ ) and once with wash buffer III ( $20 \mathrm{mM}$ Tris- $\mathrm{HCl}, \mathrm{pH}$ 7.5, $200 \mathrm{mM} \mathrm{LiCl}, 1 \mathrm{mM} \mathrm{EDTA}$ and $1 \mathrm{mM} \mathrm{DTT}$ ). Cell lysates and successive washes remained viscous, and thus bead homogenization was sometimes difficult, and bead separation (PureProteome Magnetic Stand, Millipore) took several minutes. Beads and inputs were resuspended in $2 \times$ SDS loading buffer, sonicated for $5 \mathrm{~min}$ (Diagenode Bioruptor, $30 \mathrm{~s}$ on/30 s off, medium amplitude) to reduce viscosity, boiled and analyzed by SDS-PAGE and western blotting.

Coimmunoprecipitation. HEK-293 cells coexpressing tagged interaction partners were lysed in IPLS (immunoprecipitation low salt; Tris-HCl, pH 7.5 50 mM, EDTA, pH 8, 0.5 mM, 0.5\% NP-40, 10\% glycerol, 120 mM NaCl, cOmplete Protease Inhibitor (Roche) and Halt Phosphatase Inhibitors (Thermo Scientific)). If needed, cleared cell lysates were incubated at $37^{\circ} \mathrm{C}$ for $30 \mathrm{~min}$ with or without RNase A $(10 \mu \mathrm{g} / \mathrm{ml}$ Thermo Scientific). Supernatants were then incubated with anti-FLAG M2 agarose beads (Sigma-Aldrich) or anti-HA agarose beads (Roche) for $2 \mathrm{~h}$ at $4{ }^{\circ} \mathrm{C}(15 \mu \mathrm{l}$ of beads per half of a 10 -cm culture dish). Beads were washed once with IPLS, twice with IPMS (immunoprecipitation medium salt, IPLS with $500 \mathrm{mM} \mathrm{NaCl}$ ) and once with IPLS. Immunoprecipitates were boiled in $2 \times$ SDS loading buffer and analyzed by SDS-PAGE and western blotting.

For sequential coimmunoprecipitations, cell lysates were first subjected to anti-FLAG immunoprecipitation. Immunoprecipitates were selectively eluted from anti-FLAG agarose beads by three successive competitive elutions with the $3 \times$ FLAG peptide (ApexBio) $(0.2 \mathrm{mg} / \mathrm{ml}$ in IPLS, three times for $15 \mathrm{~min}$ each at $4{ }^{\circ} \mathrm{C}$ with one volume of beads). Eluates were cleared by incubation with Protein A/G PLUS agarose beads for $2 \mathrm{~h}$ at $4{ }^{\circ} \mathrm{C}$ before the second anti-HA agarose immunoprecipitation.

For endogenous coimmunoprecipitations, HeLa cell lysates were prepared with a modified IPLS-HEPES buffer (50 mM HEPES-NaOH, pH 7.4, instead of Tris buffer), precleared with Protein G magnetic beads (Millipore) for $2 \mathrm{~h}$ at $4{ }^{\circ} \mathrm{C}$, and incubated for $3 \mathrm{~h}$ with Protein $\mathrm{G}$ magnetic beads (Millipore) and $2 \mu \mathrm{g}$ of anti-ERG antibody (sc-353 for CCR4-NOT co-IP, sc-354 for RBPMS co-IP), anti-RBPMS antibody or normal rabbit IgG (Santa-Cruz) per $15-\mathrm{cm}$ cell culture dish. Beads were washed four times with IPLS-HEPES buffer. Immunoprecipitates were boiled in $2 \times$ SDS loading buffer and analyzed by SDS-PAGE and western blotting.

High-throughput yeast-two hybrid assays. The 11 DNA fragments corresponding to the three full-length human Erg ORFs and their individualized domains (Fig. 2a) were cloned in pAD-dest-CYH and pDB-dest and transformed in MATa Y8800 and MAT $\alpha$ Y8930 Saccharomyces cerevisiae strains, respectively (described in ref. 56) as described in ref. 57. One pool of $11 \mathrm{AD}$-Erg Y8800 was mated to each of the 15,483 DB-ORFs Y 8930 of the hORFeome v5.1 (CCSB, Dana-Farber Cancer Institute) and each of our 11 DB-Erg Y9300. 165 pools of 94 AD-ORFs were mated to each of the 11 DB-Erg Y8930. A stringent Y2H screening was performed in the reciprocal orientation, strictly as described in ref. 58. Colonies positive for the GAL1::HIS3 and GAL1::ADE2 selective markers but negative for autoactivation were selected for PCR amplification and identification of interacting proteins by sequencing of the respective $\mathrm{AD}$ - and $\mathrm{DB}-\mathrm{ORF}$.

RNA immunoprecipitation (RIP). The RIP protocol was derived from ref. 59. HEK-293 cells cotransfected with FLAG-ERG, HA-RBPMS-MS2-CP and R-Luc$8 \mathrm{MS} 2$ reporter were washed four times with cold PBS and lysed 5 min on ice with 1 volume of polysome lysis buffer (PLB) $\left(100 \mathrm{mM} \mathrm{KCl}, 5 \mathrm{mM} \mathrm{MgCl}_{2}, 10 \mathrm{mM}\right.$ HEPES, pH 7.0, 0.5\% NP-40, 1 mM DTT, cOmplete Protease Inhibitor (Roche), Halt Phosphatase Inhibitors (Thermo Scientific) and Protector RNase Inhibitor (Roche)). Lysates were snap frozen at $-80^{\circ} \mathrm{C}$ and thawed on ice to complete lysis. Anti-FLAG M2 agarose beads were washed with PLB and incubated with blocking buffer (PLB, $0.1 \%$ BSA and $1 \mathrm{mg} / \mathrm{ml}$ yeast or E. coli tRNA) for $1 \mathrm{~h}$ at $4{ }^{\circ} \mathrm{C} .1 \%$ and $10 \%$ of cleared cell lysates were saved as RNA and protein inputs, respectively. Lysates were then incubated with blocked anti-FLAG M2 agarose beads for $2 \mathrm{~h}$ at $4{ }^{\circ} \mathrm{C}$. Beads were then washed four times with ice-cold NT2 buffer (50 mM Tris-HCl, pH 7.4, 150 mM NaCl, 1 mM MgCl $2,0.05 \%$ NP-40 and $1 \mathrm{mM}$ DTT). $10 \%$ of washed beads were saved for verifying (co)immunoprecipitation of FLAG-ERG and HA-RBPMS-MS2-CP. Total RNA was extracted from inputs and immunoprecipitates and processed for RT-qPCR as described above. RIP enrichment was calculated as a percentage of input of the $R$-Luc mRNA normalized to the percentage of input of the GAPDH mRNA.

For RIP of endogenous AURKA and AURKB mRNAs, ten $15-\mathrm{cm}$ dishes of $50 \%$ confluent HA-FLAG-tagged ERG, RBPMS or CNOT2 stable HeLa cell lines were induced with doxycycline to express ectopic tagged proteins at levels similar to those of endogenous proteins (5, 5 and $500 \mathrm{ng} / \mathrm{ml}$, respectively, for $24 \mathrm{~h}$ ). Induced and control HeLa cells were lysed in $10 \mathrm{ml}$ of IPLS-HEPES with $1 \mathrm{mM}$ DTT and Protector RNase Inhibitor (Roche). Cleared lysates were incubated with $50 \mu \mathrm{l}$ of anti-FLAG MS2 magnetic beads (Sigma) for $3 \mathrm{~h}$ and washed four times in IPLSHEPES complemented with $1 \mathrm{mM}$ DTT. Immunoprecipitates were selectively eluted from anti-FLAG magnetic beads by three successive competitive elutions with $3 \times$ FLAG peptide (ApexBio) $(0.2 \mathrm{mg} / \mathrm{ml}$ in IPLS-HEPES, three times for $15 \mathrm{~min}$ each at $4{ }^{\circ} \mathrm{C}$ with one volume of beads). Total RNA was extracted from inputs and immunoprecipitates, and processed for RT-qPCR as described above. Owing to low signals in immunoprecipitates, RNA was suspended in $20 \mu \mathrm{l}$ of RNase-free water, the maximum amount of RNA was used in reverse transcription reactions, and cDNA was diluted only ten-fold in the final RT-qPCR reactions. RIP enrichment was calculated as a percentage of input of the AURKA or AURKB mRNAs normalized to the percentage of input of the 18S rRNA.

Microarray analysis. siRNA-transfected cells were treated with ActD $(5 \mu \mathrm{M})$ for $0,0.5,1,2$ or $4 \mathrm{~h}$. Total RNA was extracted, and RNA quality was assessed by electrophoresis (Experion Automated Electrophoresis System, Bio-Rad). Gene expression profiling was carried out on four independent experiments with HumanHT-12 v4 Expression BeadChips (Illumina) according to standard procedures. From a total of 47,231 probe sets, 7,507 probe sets were considered to be noncoding genes and were removed on the basis of not having ' $m$ RNA' in their Illumina definition. A first-order linear regression was fitted to the logarithm of the intensity points normalized to $\mathrm{T} 0$, by following a first-order kinetics model. Three control probes (corresponding to stable housekeeping transcripts GAPDH, NDUFA12 and RPL32) were used for normalization. Only probes showing decay, i.e., those with a control slope $\leq 0(19,195$ probes corresponding to 15,532 different genes) were selected for further analysis. The areas under siCTL and siERG degradation curves were calculated, and differences in areas between siCTL and siERG were fitted on a normal distribution curve. A $P$ value was computed for each probe set. Probes with a negative or positive difference and an FDR-corrected $P$ value $<0.05$ were considered to be stabilized or more degraded, respectively, in siERG-transfected cells.

FACS labeling and analysis. Cells were trypsinized, rinsed three times with cold PBS, fixed with ice-cold $70 \%$ ethanol for $30 \mathrm{~min}$ at $-20{ }^{\circ} \mathrm{C}$, and used immediately or stored at $-20^{\circ} \mathrm{C}$. Fixed cells were permeabilized with PBS with $0.25 \%$ Triton X-100 on ice for 5 min before successive incubations with primary anti-phospho-histone H3 (Ser10) and Alexa488-conjugated secondary antibodies in PBS with $1 \%$ BSA. Cells were then treated with RNase A $(200 \mu \mathrm{g} / \mathrm{ml})$ for $30 \mathrm{~min}$ at $37^{\circ} \mathrm{C}$. Propidium iodide was added $(40 \mu \mathrm{g} / \mathrm{ml})$, and cells were immediately subjected to FACS analysis on a FACSCalibur flow cytometer 
(BD Biosciences). Mitotic cells were identified as $2 n$ cells positive for phophohistone $\mathrm{H} 3$ with CellQuest Pro (BD Biosciences).

Statistical analyses. Graph values are presented as mean \pm s.d., calculated for at least three independent experiments. The significance between means was determined with a two-tailed unpaired Student's $t$ test. Normality was verified on control decay curves with Shapiro-Wilk tests $(P<0.01)$, and variance equality was verified for each test with an $\mathrm{F}$ test. $P$-value thresholds are depicted as follows: ${ }^{*} P<0.05 ;{ }^{*} P<0.01 ;{ }^{* *} P<0.001$; NS, not significant; ND, not determined. Significance in the overlap between ERG mRNA-decay targets and RBPMS PAR-CLIP mRNA targets or ERG ChIP gene targets was determined with Pearson's chi-squared test, considering the global set 15,532 different genes with a negative decay slope in siCTL HeLa cells (described for microarray analysis above). The significance between cumulative fraction curves was calculated with a Kolmogorov-Smirnov test.
53. Lykke-Andersen, J., Shu, M.D. \& Steitz, J.A. Human Upf proteins target an mRNA for nonsense-mediated decay when bound downstream of a termination codon. Cell 103, 1121-1131 (2000).

54. Souquere, S. et al. Unravelling the ultrastructure of stress granules and associated P-bodies in human cells. J. Cell Sci. 122, 3619-3626 (2009).

55. Raj, A., van den Bogaard, P., Rifkin, S.A., van Oudenaarden, A. \& Tyagi, S. Imaging individual mRNA molecules using multiple singly labeled probes. Nat. Methods 5, 877-879 (2008).

56. James, P., Halladay, J. \& Craig, E.A. Genomic libraries and a host strain designed for highly efficient two-hybrid selection in yeast. Genetics 144, 1425-1436 (1996).

57. Walhout, A.J. \& Vidal, M. High-throughput yeast two-hybrid assays for large-scale protein interaction mapping. Methods 24, 297-306 (2001).

58. Rual, J.F. et al. Towards a proteome-scale map of the human protein-protein interaction network. Nature 437, 1173-1178 (2005).

59. Keene, J.D., Komisarow, J.M. \& Friedersdorf, M.B. RIP-Chip: the isolation and identification of mRNAs, microRNAs and protein components of ribonucleoprotein complexes from cell extracts. Nat. Protoc. 1, 302-307 (2006). 